\title{
MICROLOCAL PROPERTIES OF BISINGULAR OPERATORS
}

\author{
MASSIMO BORSERO AND RENÉ SCHULZ
}

\begin{abstract}
We study the microlocal properties of bisingular operators, a class of operators on the product of two compact manifolds. We define a wave front set for such operators, and analyse its properties. We compare our wave front set with the $S G$ wave front set, a global wave front set which shares with it formal similarities.
\end{abstract}

\section{INTRODUCTION}

Bisingular operators were originally introduced by L. Rodino in Rod75] as a class of operators on a product of two compact manifolds $\Omega_{1} \times \Omega_{2}$, defined as linear and continuous operators $A=O p(a)$ whose symbol satisfies, in local product-type coordinates, the estimate

$$
\left|D_{\xi_{1}}^{\alpha_{1}} D_{\xi_{2}}^{\alpha_{2}} D_{x_{1}}^{\beta_{1}} D_{x_{2}}^{\beta_{2}} a\left(x_{1}, x_{2}, \xi_{1}, \xi_{2}\right)\right| \leq C_{\alpha_{1}, \alpha_{2}, \beta_{1}, \beta_{2}}\left\langle\xi_{1}\right\rangle^{m_{1}-\left|\alpha_{1}\right|}\left\langle\xi_{2}\right\rangle^{m_{2}-\left|\alpha_{2}\right|} .
$$

A simple and fundamental example of a bisingular operator is the tensor product $A_{1} \otimes A_{2}$ of two pseudodifferential operators, with symbols in the Hörmander class, $A_{i} \in \mathrm{L}^{m_{i}}\left(\Omega_{i}\right), i=1,2$, while more complex examples include the vector-tensor product $A_{1} \otimes A_{2}$ studied in [Rod75], and the double Cauchy integral operator studied in [NR06]. To each symbols of a bisingular operator we can associate two maps

$$
\begin{aligned}
\sigma^{1}: \Omega_{1} \times \mathbb{R}^{n_{1}} \rightarrow \mathrm{L}^{m_{2}}\left(\Omega_{2}\right) \\
\sigma^{2}: \Omega_{2} \times \mathbb{R}^{n_{2}} \rightarrow \mathrm{L}^{m_{1}}\left(\Omega_{1}\right),
\end{aligned}
$$

and with these maps the bisingular calculus takes the form of a calculus with vector valued symbols. General vector valued calculi have been deeply studied, for example, by B. V. Fedosov, B.-W. Schulze and N. N. Tarkhanov in [FST98].

In this paper we deal with bisingular operators whose symbols follow Hörmander-type estimates (see e.g. [Hör85]), however a global version of bisingular calculus was defined by U. Battisti, T. Gramchev, S. Pilipović and L. Rodino in [BGPR13. In particular, we only study operators on compact manifolds, given explicitly in local coordinates. We also note that 'product-type' operators calculi, similar to bisingular calculus, were introduced by V. S. Pilidi [Pil73], R. V. Dudučava [Dud79a], Dud79b], and, more recently, by R. Melrose and F. Rochon [MR06]. Moreover, multisingular calculi were considered by V. S. Pilidi [Pil71] and L. Rodino Rod80].

Applications of bisingular calculus include Index Theory, see e.g. [NR06, Analitic Number Theory, see e.g. [Bat12, and Geometric Analysis, see

2010 Mathematics Subject Classification. 35S05, 35A18, 35A27.

Key words and phrases. Bisingular operators, Microlocal analysis, Wave front set. 
e.g. GH13.

The aim of this paper is to study the microlocal properties of bisingular operators. In order to do this, we define a suitable wave front set for such operators, called the Bi-wave front set, which is the union of three components

$$
\mathrm{WF}_{\mathrm{bi}}(u)=\mathrm{WF}_{\mathrm{bi}}^{1}(u) \cup \mathrm{WF}_{\mathrm{bi}}^{2}(u) \cup \mathrm{WF}_{\mathrm{bi}}^{12}(u),
$$

$u \in \mathcal{D}^{\prime}\left(\Omega_{1} \times \Omega_{2}\right)$. This definition is formulated using the calculus only, and, roughly speaking, has this interpretation: $\mathrm{WF}_{\mathrm{bi}}^{1}$ takes care of the singularities which, in the wave front space, lie in the axis $\xi_{2}=0, \mathrm{WF}_{\mathrm{bi}}^{2}$ takes care of the singularities which lie in the axis $\xi_{1}=0$, and $\mathrm{WF}_{\mathrm{bi}}^{12}$ of the remaining singularities, which include all the classical ones. Our wave front set is related to the classical Hörmander wave front set $\mathrm{WF}_{\mathrm{cl}}$ (see [Hör83]) via the following inclusion

$$
\mathrm{WF}_{\mathrm{cl}} u \cap\left(\Omega_{1} \times \Omega_{2} \times\left(\mathbb{R}^{n_{1}} \backslash\{0\}\right) \times\left(\mathbb{R}^{n_{2}} \backslash\{0\}\right)\right) \subset \mathrm{WF}_{\mathrm{bi}}^{12}(u) .
$$

Moreover, we have a global regularity result

$$
\mathrm{WF}_{\mathrm{bi}}(u)=\emptyset \Leftrightarrow u \in \mathcal{C}^{\infty}\left(\Omega_{1} \times \Omega_{2}\right),
$$

which implies that the bi-wave front set encompasses all the singularities. Our main result is the following:

Proposition 0.1. Let $C$ be a bisingular operator, $u \in \mathcal{D}^{\prime}\left(\Omega_{1} \times \Omega_{2}\right)$. Then

$$
\mathrm{WF}_{\mathrm{bi}}(C u) \subset \mathrm{WF}_{\mathrm{bi}}(u) \text {. }
$$

This Proposition shows the our wave front set is microlocal with respect to bisingular operators. Then, we define an appropriate notion of characteristic set for bisingular operators, given again as a union of three components,

$$
\operatorname{Char}_{\mathrm{bi}}(C):=\operatorname{Char}_{\mathrm{bi}}^{1}(C) \cup \operatorname{Char}_{\mathrm{bi}}^{2}(C) \cup \operatorname{Char}_{\mathrm{bi}}^{12}(C),
$$

and with this notion we can get a microellipticity result for the 1- and 2components of the bi-wave front set

Proposition 0.2. Let $C$ be a bisingular operator, $u \in \mathcal{D}^{\prime}\left(\Omega_{1} \times \Omega_{2}\right)$. Then

$$
\mathrm{WF}_{\mathrm{bi}}^{i}(u) \subseteq \operatorname{Char}_{\mathrm{bi}}^{i}(C) \cup \mathrm{WF}_{\mathrm{bi}}^{i}(C u),
$$

$i=1,2$.

We note strong formal similarities between the bisingular calculus and the so called $S G$-calculus, introduced on $\mathbb{R}^{n}$ by H. O. Cordes [Cor95] and C. Parenti [Par72], see also R. Melrose [Mel95], Y. Egorov and B.-W. Schulze [ES97, and E. Schrohe [Sch87]. For this reason, our wave front set has formal connections to and similar features as the global $S G$-wave front set, or $\mathscr{S}$-wave front set, introduced by S. Coriasco and L. Maniccia [CM03], see also R. Melrose Mel94 for a geometric scattering version.

The paper is organized as follows. In Section 11 we fix some notation and briefly review the bisingular calculus. In Section 2, following the ideas in [Hör83 and GS94, we study the mapping properties of bisingular operators and their microlocal properties with respect to the classical wave front set $\mathrm{WF}_{\mathrm{cl}}$. In Section 3 we define the bi-wave front set and state the main results concercing microlocality and microellipticity of bisingular operators. 
In Sections 4 we compare the bisingular calculus and the $S G$ calculus, focusing on the relations and differences between the bi-wave front set and the $S G$ wave front set.

Acknowledgements. We are grateful to Profs. D. Bahns, U. Battisti, S. Coriasco, L. Rodino, I. Witt for valuable advice and constructive criticism. This work was supported by the German Research Foundation (Deutsche Forschungsgemeinschaft, DFG) through the Institutional Strategy of the University of Göttingen, in particular through the research training group GRK 1493 and the Courant Research Center "Higher Order Structures in Mathematics".

The second author is also grateful for the support received by the Studienstiftung des Deutschen Volkes and the German Academic Exchange Service (DAAD), as part of this collaboration was funded within the framework of a "DAAD Doktorandenstipendium".

\section{Preliminaries}

1.1. Introduction to bisingular calculus. In this section we will recall the main definitions and properties of bisingular symbols and bisingular operators with homogeneous principal symbols. For the Hörmander pseudodifferential operators, whose tensor products provide the model example of bisingular operators, we use the notations from [Hör85. $\Omega_{i}, i=1,2$, denotes an open domain of $\mathbb{R}^{n_{i}}$.

Definition 1.1. $\mathrm{S}^{m_{1}, m_{2}}\left(\Omega_{1}, \Omega_{2}\right)$ is the set of $\mathcal{C}^{\infty}\left(\Omega_{1} \times \Omega_{2} \times \mathbb{R}^{n_{1}} \times \mathbb{R}^{n_{2}}\right)$ functions such that, for all multiindex $\alpha_{i}, \beta_{i}$ and for all compact subsets $K_{i} \subset \subset \Omega_{i}, i=1,2$, there exists a constant $C_{\alpha_{1}, \alpha_{2}, \beta_{1}, \beta_{2}, K_{1}, K_{2}}>0$ such that

$$
\left|D_{\xi_{1}}^{\alpha_{1}} D_{\xi_{2}}^{\alpha_{2}} D_{x_{1}}^{\beta_{1}} D_{x_{2}}^{\beta_{2}} a\left(x_{1}, x_{2}, \xi_{1}, \xi_{2}\right)\right| \leq C_{\alpha_{1}, \alpha_{2}, \beta_{1}, \beta_{2}, K_{1}, K_{2}}\left\langle\xi_{1}\right\rangle^{m_{1}-\left|\alpha_{1}\right|}\left\langle\xi_{2}\right\rangle^{m_{2}-\left|\alpha_{2}\right|},
$$

for all $x_{i} \in K_{i}, \xi_{i} \in \mathbb{R}^{n_{i}}$. As usual, $\langle\xi\rangle:=\left(1+|\xi|^{2}\right)^{\frac{1}{2}}$. An element of $\mathrm{S}^{m_{1}, m_{2}}\left(\Omega_{1}, \Omega_{2}\right)$ is called a symbol.

Definition 1.2. A linear operator $A: \mathcal{C}_{0}^{\infty}\left(\mathbb{R}^{n_{1}+n_{2}}\right) \rightarrow \mathcal{C}^{\infty}\left(\mathbb{R}^{n_{1}+n_{2}}\right)$ is called a bisingular operator if it can be written in the form

$$
\begin{aligned}
& A(u)\left(x_{1}, x_{2}\right)=O p(a)[u]\left(x_{1}, x_{2}\right) \\
& \quad=\frac{1}{(2 \pi)^{n_{1}+n_{2}}} \int_{\mathbb{R}^{n_{1}}} \int_{\mathbb{R}^{n_{2}}} e^{i\left(x_{1} \cdot \xi_{1}+x_{2} \cdot \xi_{2}\right)} a\left(x_{1}, x_{2}, \xi_{1}, \xi_{2}\right) \hat{u}\left(\xi_{1}, \xi_{2}\right) d \xi_{1} d \xi_{2},
\end{aligned}
$$

where $a \in \mathrm{S}^{m_{1}, m_{2}}\left(\Omega_{1}, \Omega_{2}\right)$ and $\hat{u}$ denotes the Fourier transform of $u$. $\mathrm{L}^{m_{1}, m_{2}}\left(\Omega_{1}, \Omega_{2}\right)$ denotes the set of all bisingular operators with symbol in $\mathrm{S}^{m_{1}, m_{2}}\left(\Omega_{1}, \Omega_{2}\right)$. Moreover, we set

$$
\begin{aligned}
\mathrm{S}^{\infty, \infty}\left(\Omega_{1}, \Omega_{2}\right) & :=\bigcup_{m_{1}, m_{2}} \mathrm{~S}^{m_{1}, m_{2}}\left(\Omega_{1}, \Omega_{2}\right) \\
\mathrm{S}^{-\infty,-\infty}\left(\Omega_{1}, \Omega_{2}\right) & :=\bigcap_{m_{1}, m_{2}} \mathrm{~S}^{m_{1}, m_{2}}\left(\Omega_{1}, \Omega_{2}\right)
\end{aligned}
$$

and by $\mathrm{L}^{\infty, \infty}\left(\Omega_{1}, \Omega_{2}\right), \mathrm{L}^{-\infty,-\infty}\left(\Omega_{1}, \Omega_{2}\right)$ the corresponding class of operators. The operators in $\mathrm{L}^{-\infty,-\infty}\left(\Omega_{1}, \Omega_{2}\right)$ are called smoothing operators. 
We associate to every $a \in \mathrm{S}^{m_{1}, m_{2}}\left(\Omega_{1}, \Omega_{2}\right)$ the two maps

$$
\begin{aligned}
A^{1}: & \Omega_{1} \times \mathbb{R}^{n_{1}} \rightarrow \mathrm{L}^{m_{2}}\left(\Omega_{2}\right) \\
& \left(x_{1}, \xi_{1}\right) \mapsto a\left(x_{1}, x_{2}, \xi_{1}, D_{2}\right), \\
A^{2}: & \Omega_{2} \times \mathbb{R}^{n_{2}} \rightarrow \mathrm{L}^{m_{1}}\left(\Omega_{1}\right) \\
& \left(x_{2}, \xi_{2}\right) \mapsto a\left(x_{1}, x_{2}, D_{1}, \xi_{2}\right),
\end{aligned}
$$

and for $a \in \mathrm{S}^{m_{1}, m_{2}}\left(\Omega_{1}, \Omega_{2}\right), b \in \mathrm{S}^{p_{1}, p_{2}}\left(\Omega_{1}, \Omega_{2}\right)$ we set (for fixed $x_{1}, x_{2}$ respectively)

$$
\begin{aligned}
& a \circ_{1} b\left(x_{1}, x_{2}, \xi_{1}, D_{2}\right):=\left(A^{1} \circ B^{1}\right)\left(x_{1}, x_{2}, \xi_{1}, D_{2}\right) \in \mathrm{L}^{m_{2}+p_{2}}\left(\Omega_{2}\right) \\
& a \circ_{2} b\left(x_{1}, x_{2}, D_{1}, \xi_{2}\right):=\left(A^{2} \circ B^{2}\right)\left(x_{1}, x_{2}, D_{1}, \xi_{2}\right) \in \mathrm{L}^{m_{1}+p_{1}}\left(\Omega_{1}\right) .
\end{aligned}
$$

Definition 1.3. Let $a \in \mathrm{S}^{m_{1}, m_{2}}\left(\Omega_{1}, \Omega_{2}\right)$. Then $a$ has a homogeneous principal symbol if

i) there exists $a_{m_{1} ;} \in \mathrm{S}^{m_{1}, m_{2}}\left(\Omega_{1}, \Omega_{2}\right)$ such that

$$
\begin{aligned}
a_{m_{1} ; \cdot}\left(x_{1}, x_{2}, t \xi_{1}, \xi_{2}\right) & =t^{m_{1}} a_{m_{1} ; \cdot}\left(x_{1}, x_{2}, \xi_{1}, \xi_{2}\right), \forall x_{1}, x_{2}, \xi_{2}, \forall\left|\xi_{1}\right|>1, t>0 \\
\quad a-\psi_{1}\left(\xi_{1}\right) a_{m_{1} ;} \cdot & \in \mathrm{S}^{m_{1}-1, m_{2}}
\end{aligned}
$$

where $\psi_{1}$ is an 0 -excision function. Moreover, $a_{m_{1} ;} \cdot\left(x_{1}, x_{2}, \xi_{1}, D_{2}\right) \in$ $\mathrm{L}_{\mathrm{cl}}^{m_{2}}\left(\Omega_{2}\right)$, so, being a classical symbol on $\Omega_{2}$, it admits an asymptotic expansion with respect to the $\xi_{2}$ variable.

ii) there exists $a_{: ; m_{2}} \in \mathrm{S}^{m_{1}, m_{2}}\left(\Omega_{1}, \Omega_{2}\right)$ such that

$$
\begin{aligned}
a_{\cdot ; m_{2}}\left(x_{1}, x_{2}, \xi_{1}, t \xi_{2}\right) & =t^{m_{2}} a_{\cdot ; m_{2}}\left(x_{1}, x_{2}, \xi_{1}, \xi_{2}\right), \forall x_{1}, x_{2}, \xi_{1}, \forall\left|\xi_{2}\right|>1, t>0 \\
a-\psi_{2}\left(\xi_{2}\right) a_{\cdot ; m_{2}} & \in \mathrm{S}^{m_{1}, m_{2}-1}, \psi_{2} \text { as } \psi_{1} \text { above. }
\end{aligned}
$$

Moreover, $a_{: ; m_{2}}\left(x_{1}, x_{2}, D_{1}, \xi_{2}\right) \in \mathrm{L}_{\mathrm{cl}}^{m_{1}}\left(\Omega_{1}\right)$, so, being a classical symbol on $\Omega_{1}$, it admits an asymptotic expansion with respect to the $\xi_{1}$ variable.

iii) the symbols $a_{m_{1} ;}$. and $a_{; ; m_{2}}$ have the same leading term, so there exists $a_{m_{1} ; m_{2}}$ such that

$$
\begin{aligned}
& a_{m_{1} ; \cdot}-\psi_{2}\left(\xi_{2}\right) a_{m_{1} ; m_{2}} \in \mathrm{S}^{m_{1}, m_{2}-1}\left(\Omega_{1}, \Omega_{2}\right), \\
& a_{\cdot ; m_{2}}-\psi_{1}\left(\xi_{1}\right) a_{m_{1} ; m_{2}} \in \mathrm{S}^{m_{1}-1, m_{2}}\left(\Omega_{1}, \Omega_{2}\right),
\end{aligned}
$$

and

$$
a-\psi_{1} a_{m_{1} ; \cdot}-\psi_{2} a_{\cdot ; m_{2}}+\psi_{1} \psi_{2} a_{m_{1} ; m_{2}} \in \mathrm{S}^{m_{1}-1, m_{2}-1}\left(\Omega_{1}, \Omega_{2}\right) .
$$

The symbols which admit a full bi-homogeneous expansion in $\xi_{1}$ and $\xi_{2}$ are called classical symbols, their class is denoted by $\mathrm{S}_{\mathrm{cl}}^{m_{1}, m_{2}}\left(\Omega_{1}, \Omega_{2}\right)$, and the corresponding operator class by $\mathrm{L}_{\mathrm{cl}}^{m_{1}, m_{2}}\left(\Omega_{1}, \Omega_{2}\right)$. 
The previous Definition implies that, given $A \in \mathrm{L}_{\mathrm{cl}}^{m_{1}, m_{2}}\left(\Omega_{1}, \Omega_{2}\right)$, we can define maps $\sigma^{1}, \sigma^{2}, \sigma^{12}$ in this way

$$
\begin{aligned}
\sigma^{1}(A): & T^{*} \Omega_{1} \backslash 0 \rightarrow \mathrm{L}_{\mathrm{cl}}^{m_{2}}\left(\Omega_{2}\right) \\
& \left(x_{1}, \xi_{1}\right) \mapsto a_{m_{1} ; \cdot}\left(x_{1}, x_{2}, \xi_{1}, D_{2}\right), \\
\sigma^{2}(A): & T^{*} \Omega_{2} \backslash 0 \rightarrow \mathrm{L}_{\mathrm{cl}}^{m_{1}}\left(\Omega_{1}\right) \\
& \left(x_{2}, \xi_{2}\right) \mapsto a_{\cdot ; m_{2}}\left(x_{1}, x_{2}, D_{1}, \xi_{2}\right), \\
\sigma^{12}(A): & \left(T^{*} \Omega_{1} \backslash 0\right) \times\left(T^{*} \Omega_{2} \backslash 0\right) \rightarrow \mathbb{C} \\
& \left(x_{1}, x_{2}, \xi_{1}, \xi_{2}\right) \mapsto a_{m_{1} ; m_{2}}\left(x_{1}, x_{2}, \xi_{1}, \xi_{2}\right),
\end{aligned}
$$

such that, denoting by $\sigma(P)(x, \xi)$ the principal symbol of a pseudodifferential operator $P$, we have

$$
\begin{aligned}
\sigma\left(\sigma^{1}(A)\left(x_{1}, \xi_{1}\right)\right)\left(x_{2}, \xi_{2}\right) & =\sigma\left(\sigma^{2}(A)\left(x_{2}, \xi_{2}\right)\right)\left(x_{1}, \xi_{1}\right) \\
& =\sigma^{12}(A)\left(x_{1}, x_{2}, \xi_{1}, \xi_{2}\right)=a_{m_{1} ; m_{2}}\left(x_{1}, x_{2}, \xi_{1}, \xi_{2}\right) .
\end{aligned}
$$

We call the couple $\left(\sigma^{1}(A), \sigma^{2}(A)\right)$ the principal symbol of $A$.

In the following, we only consider bisingular operators on the product of two compact manifolds $\Omega_{1}, \Omega_{2}$. They are defined as above in local coordinates. For those, there exists a notion of ellipticity, called bi-ellipticity. For more details, see [Rod75].

Definition 1.4. Let $A \in \mathrm{L}_{\mathrm{cl}}^{m_{1}, m_{2}}\left(\Omega_{1}, \Omega_{2}\right)$. We say that $A$ is bi-elliptic if

i) $\sigma^{12}(A)\left(v_{1}, v_{2}\right) \neq 0$ for all $\left(v_{1}, v_{2}\right) \in\left(T^{*} \Omega_{1} \backslash 0\right) \times\left(T^{*} \Omega_{2} \backslash 0\right)$;

ii) $\sigma^{1}(A)\left(v_{1}\right)$ is exactly invertible as an operator in $\mathrm{L}_{\mathrm{cl}}^{m_{2}}\left(\Omega_{2}\right)$ for all $v_{1} \in T^{*} \Omega_{1} \backslash 0$, with inverse in $\mathrm{L}_{\mathrm{cl}}^{-m_{2}}\left(\Omega_{2}\right)$;

iii) $\sigma^{2}(A)\left(v_{2}\right)$ is exactly invertible as an operator in $\mathrm{L}_{\mathrm{cl}}^{m_{1}}\left(\Omega_{1}\right)$ for all $v_{2} \in T^{*} \Omega_{2} \backslash 0$, with inverse in $\mathrm{L}_{\mathrm{cl}}^{-m_{1}}\left(\Omega_{1}\right)$.

To elaborate on this definition, we give some examples.

Example 1.5. Consider the differential operator

$$
A=\sum_{\substack{\left|\beta_{1}\right| \leq m_{1} \\ \beta_{2} \mid \leq m_{2}}} c_{\beta_{1}, \beta_{2}}\left(x_{1}, x_{2}\right) D_{1}^{\beta_{1}} D_{2}^{\beta_{2}},
$$

with $\mathcal{C}^{\infty}$ coefficients. In this case

$$
\begin{aligned}
\sigma^{1}(A)\left(x_{1}, \xi_{1}\right) & =\sum_{\substack{\left|\beta_{1}\right|=m_{1} \\
\left|\beta_{2}\right| \leq m_{2}}} c_{\beta_{1}, \beta_{2}}\left(x_{1}, x_{2}\right) \xi_{1}^{\beta_{1}} D_{2}^{\beta_{2}} \\
\sigma^{2}(A)\left(x_{2}, \xi_{2}\right) & =\sum_{\substack{\left|\beta_{1}\right| \leq m_{1} \\
\left|\beta_{2}\right|=m_{2}}} c_{\beta_{1}, \beta_{2}}\left(x_{1}, x_{2}\right) D_{1}^{\beta_{1}} \xi_{2}^{\beta_{2}} .
\end{aligned}
$$

A full bi-homogeneous expansion is given by

$$
\tilde{\sigma}^{i, j}(A)\left(x_{1}, x_{2}, \xi_{1}, \xi_{2}\right)=\sum_{\substack{\left|\beta_{1}\right|=i \\ \beta_{2} \mid=j}} c_{\beta_{1}, \beta_{2}}\left(x_{1}, x_{2}\right) \xi_{1}^{\beta_{1}} \xi_{2}^{\beta_{2}} .
$$

The bi-ellipticity of $A$ is given by the condition $\sigma^{12}(A)=$ $\tilde{\sigma}^{m_{1}, m_{2}}(A)\left(v_{1}, v_{2}\right) \neq 0$ for all $\left(v_{1}, v_{2}\right) \in\left(T^{*} \Omega_{1} \backslash 0\right) \times\left(T^{*} \Omega_{2} \backslash 0\right)$ and 
the invertibility of the two maps (1) and (2).

We may give a global meaning to $A$ on a product of compact manifolds $\Omega_{1} \times \Omega_{2}$ by taking, for example, $\Omega_{j}=\mathbb{T}_{j}$, the $n_{j}$-dimensional torus, $j=1,2$, and $x_{j}$ angular coordinates on $\mathbb{T}_{j}$.

With this in mind, it is possible to study some model cases of operators of the form $A \otimes B$. In the following Table 1.1 we mean by $\Psi$ DO the classical pseudodifferential operators on $\Omega_{1} \times \Omega_{2}$, and by $\Psi$ DO-order and $\Psi$ DO-ell. their order and ellipticity, respectively.

\begin{tabular}{|c|c|c|c|c|}
\hline Operator & $\Psi$ DO-order & $\Psi$ DO-ell. & Bi-order & Bi-ell. \\
\hline$I \otimes I$ & 0 & $\sqrt{ }$ & $(0,0)$ & $\sqrt{ }$ \\
\hline$-\Delta_{1} \otimes I+I \otimes\left(-\Delta_{2}\right)$ & 2 & $\sqrt{ }$ & $(2,2)$ & $\times$ \\
\hline$-\Delta_{1} \otimes\left(-\Delta_{2}\right)$ & 4 & $\times$ & $(2,2)$ & $\times$ \\
\hline$-\Delta_{1} \otimes\left(-\Delta_{2}+I\right)$ & 4 & $\times$ & $(2,2)$ & $\times$ \\
\hline$\left(-\Delta_{1}+I\right) \otimes\left(-\Delta_{2}+I\right)$ & 4 & $\times$ & $(2,2)$ & $\sqrt{ }$ \\
\hline$\left(-\Delta_{1}+I\right)^{-1} \otimes\left(-\Delta_{2}+I\right)^{-1}$ & not a $\Psi$ DO & & $(-2,-2)$ & $\sqrt{ }$ \\
\hline
\end{tabular}

TABLE 1. Some model cases of bisingular operators of tensor product type

Theorem 1.6. Let $A \in \mathrm{L}_{\mathrm{cl}}^{m_{1}, m_{2}}\left(\Omega_{1}, \Omega_{2}\right)$ be bi-elliptic. Then there exists $B \in \mathrm{L}_{\mathrm{cl}}^{-m_{1},-m_{2}}\left(\Omega_{1}, \Omega_{2}\right)$ such that

$$
\begin{aligned}
& A B=I+K_{1} \\
& B A=I+K_{2},
\end{aligned}
$$

where $I$ is the identity map and $K_{1}, K_{2}$ are smoothing operators. Moreover the principal symbol of $B$ is $\left(\sigma^{1}(A)^{-1}, \sigma^{2}(A)^{-1}\right)$.

From now on we will assume, for simplicity, that symbols of bisingular operators have compact support in the $x_{1}, x_{2}$ variables.

Theorem 1.7. Let $a \in \mathrm{S}^{m_{1}, m_{2}}\left(\Omega_{1}, \Omega_{2}\right), b \in \mathrm{S}^{p_{1}, p_{2}}\left(\Omega_{1}, \Omega_{2}\right)$. Then $A B \in$ $\mathrm{L}^{m_{1}+p_{1}, m_{2}+p_{2}}\left(\Omega_{1}, \Omega_{2}\right)$, and its symbol $c\left(x_{1}, x_{2}, \xi_{1}, \xi_{2}\right)$ has the asymptotic expansion

$$
c \sim \sum_{j=0}^{\infty} c_{m_{1}+p_{1}-j, m_{2}+p_{2}-j}
$$

where

$$
\begin{aligned}
c_{m_{1}+p_{1}-j, m_{2}+p_{2}-j}= & c_{m_{1}+p_{1}-j-1, m_{2}+p_{2}-j}^{1}+c_{m_{1}+p_{1}-j, m_{2}+p_{2}-j-1}^{2} \\
& +c_{m_{1}+p_{1}-j, m_{2}+p_{2}-j}^{12}
\end{aligned}
$$


and

$$
\begin{aligned}
& c^{1} m_{1}+p_{1}-j-1, m_{2}+p_{2}-j \\
& =\sum_{\left|\alpha_{2}\right|=j} \frac{1}{\alpha_{2} !}\left\{\partial_{\xi_{2}}^{\alpha_{2}} a \circ_{1} D_{x_{2}}^{\alpha_{2}} b-\sum_{\left|\alpha_{1}\right| \leq j} \frac{1}{\alpha_{1} !} \partial_{\xi_{1}}^{\alpha_{1}} \partial_{\xi_{2}}^{\alpha_{2}} a D_{x_{1}}^{\alpha_{1}} D_{x_{2}}^{\alpha_{2}} b\right\} \\
& c^{2}{ }_{m_{1}+p_{1}-j, m_{2}+p_{2}-j-1} \\
& =\sum_{\left|\alpha_{1}\right|=j} \frac{1}{\alpha_{1} !}\left\{\partial_{\xi_{1}}^{\alpha_{1}} a \circ_{2} D_{x_{1}}^{\alpha_{1}} b-\sum_{\left|\alpha_{2}\right| \leq j} \frac{1}{\alpha_{2} !} \partial_{\xi_{1}}^{\alpha_{1}} \partial_{\xi_{2}}^{\alpha_{2}} a D_{x_{1}}^{\alpha_{1}} D_{x_{2}}^{\alpha_{2}} b\right\} \\
& \quad=\sum_{\left|\alpha_{1}\right|=\left|\alpha_{2}\right|=j, m_{2}+p_{2}-j} \frac{1}{\alpha_{1} ! \alpha_{2} !} \partial_{\xi_{1}}^{\alpha_{1}} \partial_{\xi_{2}}^{\alpha_{2}} a D_{x_{1}}^{\alpha_{1}} D_{x_{2}}^{\alpha_{2}} b
\end{aligned}
$$

Corollary 1.7.1. Let $a \in \mathrm{S}^{m_{1}, m_{2}}\left(\Omega_{1}, \Omega_{2}\right), b \in \mathrm{S}^{p_{1}, p_{2}}\left(\Omega_{1}, \Omega_{2}\right)$. Then the commutator $[A, B]:=A B-B A$ belongs to $L^{m_{1}+p_{1}-1, m_{2}+p_{2}}+L^{m_{1}+p_{1}, m_{2}+p_{2}-1}$.

Proof. By Theorem 1.7 we have as leading order terms $(j=0)$ :

$$
\begin{aligned}
& c_{m_{1}+p_{1}-1, m_{2}+p_{2}}^{1}=a \circ_{1} b-b \circ_{1} a \\
& c_{m_{1}+p_{1}, m_{2}+p_{2}-1}^{2}=a \circ_{2} b-b \circ_{2} a \\
& c_{m_{1}+p_{1}, m_{2}+p_{2}}^{12}=0 .
\end{aligned}
$$

Then, expanding $c^{1}$ and $c^{2}$ according to the definition of $o_{j}, j=1,2$, we get

$$
c^{j}=i\{a, b\}_{j}+\text { terms of order }\left(m_{j}+p_{j}-2\right),
$$

where with $\{a, b\}_{j}$ we denote the Poisson bracket of $a$ and $b$ in the $j$ argument. Therefore, the leading terms (up to order $\left(m_{1}+p_{1}-2, m_{2}+p_{2}-2\right)$ ) of the expansion of $c$ can be written as

$$
c=0+i\left(\{a, b\}_{1}+\{a, b\}_{2}\right) \in \mathrm{S}^{m_{1}+p_{1}-1, m_{2}+p_{2}}+\mathrm{S}^{m_{1}+p_{1}, m_{2}+p_{2}-1} .
$$

Remark 1.8. This behaviour under commutators is indeed something peculiar about bisingular calculus. It has the consequence that we can not use a lot of the common "commutator tricks" in the proofs to obtain microlocal properties.

Example 1.9. For a better understanding of this phenomenon, consider the model case of a tensor product where $A=A_{1} \otimes A_{2} \in \mathrm{L}^{m_{1}, m_{2}}, B=$ $B_{1} \otimes B_{2} \in \mathrm{L}^{p_{1}, p_{2}}$. Then

$$
\begin{aligned}
{[A, B]=\left[A_{1} \otimes A_{2}, B_{1} \otimes B_{2}\right] } & =A_{1} B_{1} \otimes A_{2} B_{2}-B_{1} A_{1} \otimes B_{2} A_{2} \\
& =\underbrace{\left[A_{1}, B_{1}\right] \otimes A_{2} B_{2}}_{\mathrm{L}^{m_{1}+p_{1}-1, m_{2}+p_{2}}}+\underbrace{B_{1} A_{1} \otimes\left[A_{2}, B_{2}\right]}_{\mathrm{L}^{m_{1}+p_{1}, m_{2}+p_{2}-1}} .
\end{aligned}
$$

To close this section, we note that all pseudodifferential operators of order zero or lower, in particular those corresponding to cut-offs and excision functions, are bisingular operators.

Lemma 1.9.1. $\mathrm{S}^{0}\left(\Omega_{1} \times \Omega_{2}\right) \subset \mathrm{S}^{0,0}\left(\Omega_{1}, \Omega_{2}\right)$. 
Proof. Let $a \in \mathrm{S}^{0}\left(\Omega_{1} \times \Omega_{2}\right)$. Then for all pair of multiindex $\alpha=\left(\alpha_{1}, \alpha_{2}\right), \beta=$ $\left(\beta_{1}, \beta_{2}\right)$ we have

$$
\begin{aligned}
\left|D_{\xi_{1}}^{\alpha_{1}} D_{\xi_{2}}^{\alpha_{2}} D_{x_{1}}^{\beta_{1}} D_{x_{2}}^{\beta_{2}} a\left(x_{1}, x_{2}, \xi_{1}, \xi_{2}\right)\right| & =\left|D_{\xi}^{\alpha} D_{x}^{\beta} a(x, \xi)\right| \prec\langle\xi\rangle^{-|\alpha|} \\
& \prec\langle\xi\rangle^{-\left|\alpha_{1}\right|}\langle\xi\rangle^{-\left|\alpha_{2}\right|} \prec\left\langle\xi_{1}\right\rangle^{-\left|\alpha_{1}\right|}\left\langle\xi_{2}\right\rangle^{-\left|\alpha_{2}\right|},
\end{aligned}
$$

that is $a \in \mathrm{S}^{0,0}\left(\Omega_{1}, \Omega_{2}\right)$.

\section{Classical microlocal analysis of Bisingular operators}

Let us first recall the notion of classical wave front set, as introduced by Hörmander [Hör83].

Definition 2.1. Let $\Omega \subset \mathbb{R}^{n}$ open, $u \in \mathcal{D}^{\prime}(\Omega)$. The distribution $u$ is microlocally $\mathcal{C}^{\infty}$ near $\left(x_{0}, \xi_{0}\right) \in \mathrm{T}^{*} \Omega \backslash 0$ if one of the following equivalent conditions is satisfied:

(1) There exists a pseudodifferential operator $A \in \mathrm{L}^{0}(\Omega)$, noncharacteristic at $\left(x_{0}, \xi_{0}\right)$, such that $A u \in \mathcal{C}^{\infty}(\Omega)$,

(2) There exists a cut-off $\phi \in \mathcal{C}_{0}^{\infty}(\Omega)$ with $\phi \equiv 1$ in an open set containing $x_{0}$ such that there exists a conic open set $\Gamma \subset \mathbb{R}^{n} \backslash 0$ containing $\xi_{0}$ and constants $C_{N}, R>0$ such that $\forall N \in \mathbb{N}$

$$
|\widehat{\phi u}(\xi)| \leq C_{N}\langle\xi\rangle^{-N} \quad \forall \xi \in \Gamma,|\xi|>R
$$

The classical wave front set of $u \in \mathcal{D}^{\prime}(\Omega)$, that we denote by $\mathrm{WF}_{\mathrm{cl}}(u)$, is the complement of the set of points where $u$ is microlocally $\mathcal{C}^{\infty}$.

It is now interesting to compare, for given operators $A$, the sets $\mathrm{WF}_{\mathrm{cl}}(A u)$ and $\mathrm{WF}_{\mathrm{cl}}(u)$. An operator is microlocal, if $\mathrm{WF}_{\mathrm{cl}}(A u) \subset \mathrm{WF}_{\mathrm{cl}}(u)$.

2.1. Mapping properties of bisingular operators. In this Section we shall estimate the classical wave front set $\mathrm{WF}_{\mathrm{cl}}(A u)$ for a linear operator $A$ and a distribution $u$ in terms of the Schwartz kernel $K_{A}$ of $A$, defined as follows:

Definition 2.2. To each operator $A: \mathcal{D}\left(\Omega_{1}\right) \rightarrow \mathcal{D}^{\prime}\left(\Omega_{2}\right)$ we can uniquely associate a distribution $K_{A}$, called the Schwartz kernel of $A$, such that for all $f \in \mathcal{D}\left(\Omega_{1}\right), g \in \mathcal{D}\left(\Omega_{2}\right)$ we have

$$
\langle A f, g\rangle=\left\langle K_{A}, f \otimes g\right\rangle .
$$

The Schwartz kernel of a bisingular operator with symbol $a$ is then defined by the oscillatory integral

(3) $K_{A}\left(x_{1}, x_{2}, y_{1}, y_{2}\right)=\int_{\mathbb{R}^{n_{1}+n_{2}}} e^{i\left(x_{1}-y_{1}, x_{2}-y_{2}\right) \cdot\left(\xi_{1}, \xi_{2}\right)} a\left(x_{1}, x_{2}, \xi_{1}, \xi_{2}\right) d \xi_{1} d \xi_{2}$.

The Schwartz kernel Theorem states the following smoothing property:

Proposition 2.3. A linear map $\mathcal{D}^{\prime}\left(\Omega_{1} \times \Omega_{2}\right) \rightarrow \mathcal{D}^{\prime}\left(\Omega_{1} \times \Omega_{2}\right)$ is actually a mapping to $\mathcal{D}\left(\Omega_{1} \times \Omega_{2}\right)$, i.e. smoothing, if and only if its distributional kernel is in $\mathcal{D}\left(\left(\Omega_{1} \times \Omega_{2}\right)^{\times 2}\right)$.

Therefore pseudodifferential operators with symbols in $S^{-\infty}$ and bisingular operators with symbol in $S^{-\infty,-\infty}$ can be seen to be smoothing. 
As the prototype of a bisingular operator is the tensor product of two pseudodifferential operators, it makes sense also to define what is meant by an operator that is smoothing in one set of variables only.

Definition 2.4. We define the space $\mathcal{C}^{\infty}\left(\Omega_{1}, \mathcal{D}^{\prime}\left(\Omega_{2}\right)\right)$ as all such $u \in \mathcal{D}^{\prime}\left(\Omega_{1} \times\right.$ $\left.\Omega_{2}\right)$ such that for each $f \in \mathcal{D}\left(\Omega_{2}\right)$, the distribution $\mathcal{D}\left(\Omega_{1}\right) \ni u(g \otimes \cdot): g \mapsto$ $u(g \otimes f)$ is actually a smooth function.

Correspondingly, we can define $\mathcal{C}^{\infty}\left(\Omega_{2}, \mathcal{D}^{\prime}\left(\Omega_{1}\right)\right)$.

We can now list the mapping properties of bisingular operators on these spaces, following the ideas in [Trè67].

Lemma 2.4.1. Bisingular operators map the spaces $\mathcal{C}^{\infty}\left(\Omega_{1}, \mathcal{D}^{\prime}\left(\Omega_{2}\right)\right)$ and $\mathcal{C}^{\infty}\left(\Omega_{2}, \mathcal{D}^{\prime}\left(\Omega_{1}\right)\right)$ into themselves.

Let $a \in S^{-\infty, m}$, then the bisingular operator $O p(a)$ maps $\mathcal{D}^{\prime}\left(\Omega_{1} \times \Omega_{2}\right)$ to $\mathcal{C}^{\infty}\left(\Omega_{1}, \mathcal{D}^{\prime}\left(\Omega_{2}\right)\right)$ and $\mathcal{C}^{\infty}\left(\Omega_{2}, \mathcal{D}^{\prime}\left(\Omega_{1}\right)\right)$ to $\mathcal{C}^{\infty}\left(\Omega_{1} \times \Omega_{2}\right)$.

Accordingly, let $a \in S^{m,-\infty}$, then the bisingular operator $O p(a)$ maps $\mathcal{D}^{\prime}\left(\Omega_{1} \times \Omega_{2}\right)$ to $\mathcal{C}^{\infty}\left(\Omega_{2}, \mathcal{D}^{\prime}\left(\Omega_{1}\right)\right)$ and $\mathcal{C}^{\infty}\left(\Omega_{1}, \mathcal{D}^{\prime}\left(\Omega_{2}\right)\right)$ to $\mathcal{C}^{\infty}\left(\Omega_{1} \times \Omega_{2}\right)$.

The following Lemma (see e.g. [GS94]) indicates how the singularities of a distribution transform under the action of a linear operator in terms of the singularities of its kernel.

Lemma 2.4.2. Let $K \subset \mathcal{D}^{\prime}\left(\Omega_{1} \times \Omega_{2}\right)$, and denote by the same letter the corresponding operator $K: \mathcal{C}_{0}^{\infty}\left(\Omega_{2}\right) \rightarrow \mathcal{D}^{\prime}\left(\Omega_{1}\right)$. Set

$$
\begin{aligned}
& \mathrm{WF}^{\prime}(K):=\left\{\left(x_{1}, x_{2}, \xi_{1},-\xi_{2}\right) \in T^{*}\left(\Omega_{1} \times \Omega_{2}\right) \backslash 0 ;\left(x_{1}, x_{2}, \xi_{1}, \xi_{2}\right) \in \mathrm{WF}_{\mathrm{cl}}(K)\right\} \\
& \mathrm{WF}_{\Omega_{1}}^{\prime}(K):=\left\{\left(x_{1}, \xi_{1}\right) \in T^{*} \Omega_{1} \backslash 0 ; \exists y \in \Omega_{2} \operatorname{with}\left(x_{1}, y, \xi_{1}, 0\right) \in \mathrm{WF}^{\prime}(K)\right\} \\
& \mathrm{WF}_{\Omega_{2}}^{\prime}(K):=\left\{\left(x_{2}, \xi_{2}\right) \in T^{*} \Omega_{2} \backslash 0 ; \exists x \in \Omega_{1} \operatorname{with}\left(x, x_{2}, 0, \xi_{2}\right) \in \mathrm{WF}^{\prime}(K)\right\} .
\end{aligned}
$$

Then if $u \in \mathcal{E}^{\prime}\left(\Omega_{2}\right)$ and $\mathrm{WF}_{\mathrm{cl}}(u) \cap \mathrm{WF}_{\Omega_{2}}^{\prime}(K)=\emptyset$, we have

$$
\mathrm{WF}_{\mathrm{cl}}(K u) \subset \mathrm{WF}^{\prime}(K)\left(\mathrm{WF}_{\mathrm{cl}}(u)\right) \cup \mathrm{WF}_{\Omega_{1}}^{\prime}(K),
$$

where we considered $\mathrm{WF}^{\prime}(K)$ as a relation $T^{*} \Omega_{2} \rightarrow T^{*} \Omega_{1}$.

2.2. Classical microlocality properties of bisingular operators. From the previous Lemma it is easy to derive that all pseudodifferential operators are microlocal. In fact, if $K$ is the kernel of a pseudodifferential operator $A$ on $\Omega=\Omega_{1}=\Omega_{2}$, then $\mathrm{WF}_{\Omega_{1}}^{\prime}(K)=\emptyset=\mathrm{WF}_{\Omega_{2}}^{\prime}(K), \mathrm{WF}^{\prime}(K)=$ $\{(x, x, \xi,-\xi)\}$, hence from Lemma 2.4.2 we get $\mathrm{WF}_{\mathrm{cl}}(A u) \subset \mathrm{WF}_{\mathrm{cl}}(u)$. We now study the microlocal properties of bisingular operators.

Example 2.5. Consider $\Omega_{1}=\Omega_{2}=\mathbb{R}$. We further pick positive $\phi, \psi \in$ $\mathcal{C}_{0}^{\infty}(\mathbb{R})$. Now define the pseudodifferential operator $T_{\phi}$ on $\mathcal{C}_{0}^{\infty}(\mathbb{R})$ by

$$
T_{\phi}(f):=\phi * f .
$$

Then the operator $A:=T_{\phi} \otimes I$ is a tensor product of two pseudodifferential operators and thus a bisingular operator on $\mathbb{R}^{2}$.

Now consider the distribution $u=\psi \otimes \delta$. It has the following wave front set:

$$
\mathrm{WF}_{\mathrm{cl}}(u)=\left\{\left(x_{1}, 0,0, \xi_{2}\right) \mid x_{1} \in \operatorname{supp}(\psi), \xi_{2} \in \mathbb{R} \backslash 0\right\} .
$$

Then it is easy to see that

$$
\mathrm{WF}_{\mathrm{cl}}(A u)=\left\{\left(x_{1}, 0,0, \xi_{2}\right) \mid x_{1} \in(\operatorname{supp}(\psi)+\operatorname{supp}(\phi)), \xi_{2} \in \mathbb{R} \backslash 0\right\} .
$$


The example can be similarly given in local coordinates on a product of two compact manifolds. We restricted ourselves to the Euclidean space for the sake of comprehensibility.

The previous example shows that, in general, bisingular operators do not have the microlocal property. As a motivation, we start with the model case of a tensor product of two pseudodifferential operators. For that we use the well-known estimate for the wave front set of a tensor product of distributions (cf. e.g. Hör83):

Lemma 2.5.1. Let $u \in \mathcal{D}^{\prime}\left(\Omega_{1}\right), v \in \mathcal{D}^{\prime}\left(\Omega_{2}\right)$. Then

$$
\begin{gathered}
\mathrm{WF}_{\mathrm{cl}}(u \otimes v) \subset \mathrm{WF}_{\mathrm{cl}}(u) \times \mathrm{WF}_{\mathrm{cl}}(v) \cup(\operatorname{supp}(u) \times\{0\}) \times \mathrm{WF}_{\mathrm{cl}}(v) \\
\cup \mathrm{WF}_{\mathrm{cl}}(v) \times(\operatorname{supp}(v) \times\{0\}) .
\end{gathered}
$$

This can be used to estimate the wave front relation for a bisingular operator given as the tensor product of two pseudodifferential operators. As a matter of fact this extends by direct calculation using standard techniques of integral regularization (see, e.g. Hör83, Shu01, GS94]) to

Theorem 2.6. Let $A \in \mathrm{L}^{m_{1}, m_{2}}\left(\Omega_{1}, \Omega_{2}\right)$. Then we can estimate the wave front set of the corresponding kernel as follows:

$$
\begin{aligned}
\mathrm{WF}_{\mathrm{cl}}\left(\mathcal{K}_{A}\right) & \subset\left\{\left(x_{1}, x_{2}, x_{1}, x_{2}, \xi_{1}, \xi_{2},-\xi_{1},-\xi_{2}\right) \mid x_{i} \in \Omega_{i}, \xi_{i} \in \mathbb{R}^{n_{i}} \backslash 0\right\} \\
& \cup\left\{\left(x_{1}, x_{2}, x_{1}, y_{2}, \xi_{1}, 0,-\xi_{1}, 0\right) \mid x_{i}, y_{i} \in \Omega_{i}, \xi_{1} \in \mathbb{R}^{n_{1}} \backslash 0\right\} \\
& \cup\left\{\left(x_{1}, x_{2}, y_{1}, x_{2}, 0, \xi_{2}, 0,-\xi_{2}\right) \mid x_{i}, y_{i} \in \Omega_{i}, \xi_{2} \in \mathbb{R}^{n_{2}} \backslash 0\right\} .
\end{aligned}
$$

Corollary 2.6.1. Let $A \in \mathrm{L}^{m_{1}, m_{2}}\left(\Omega_{1}, \Omega_{2}\right), u \in \mathcal{E}^{\prime}\left(\Omega_{1} \times \Omega_{2}\right)$. Then

$$
\begin{aligned}
\mathrm{WF}_{\mathrm{cl}}(A u) \subset \mathrm{WF}_{\mathrm{cl}}(u) \cup\left\{\left(x_{1}, x_{2}, 0, \xi_{2}\right) ; \exists y_{1} \in \Omega_{1}:\left(y_{1}, x_{2}, 0, \xi_{2}\right) \in \mathrm{WF}_{\mathrm{cl}}(u)\right\} \\
\cup\left\{\left(x_{1}, x_{2}, \xi_{1}, 0\right) ; \exists y_{2} \in \Omega_{2}:\left(x_{1}, y_{2}, \xi_{1}, 0\right) \in \mathrm{WF}_{\mathrm{cl}}(u)\right\} .
\end{aligned}
$$

Proof. Use Theorem 2.6 and Lemma 2.4.2.

Based on this observation we find that bisingular operators are microlocal with respect to a modified version of the classical wave front set. It is obtained by dropping the information about the precise location of the singularities with the covariable $\xi_{1}=0$ or $\xi_{2}=0$ in the corresponding variable.

Proposition 2.7. Let $A \in \mathrm{L}^{m_{1}, m_{2}}\left(\Omega_{1}, \Omega_{2}\right), u \in \mathcal{E}^{\prime}\left(\Omega_{1} \times \Omega_{2}\right)$. Then

$$
\begin{aligned}
\mathrm{WF}_{\mathrm{cl}}(A u) & \subset \widetilde{\mathrm{WF}_{\mathrm{cl}}}(u) \\
& :=\left\{\left(x_{1}, x_{2}, \xi_{1}, \xi_{2}\right):\left(x_{1}, x_{2}, \xi_{1}, \xi_{2}\right) \in \mathrm{WF}_{\mathrm{cl}}(u) ;\left|\xi_{1}\right|\left|\xi_{2}\right| \neq 0\right\} \\
& \cup\left\{\left(x_{1}, x_{2}, \xi_{1}, 0\right) ; \exists y_{2} \in \Omega_{2}:\left(x_{1}, y_{2}, \xi_{1}, 0\right) \in \mathrm{WF}_{\mathrm{cl}}(u)\right\} \\
& \cup\left\{\left(x_{1}, x_{2}, 0, \xi_{2}\right) ; \exists y_{1} \in \Omega_{1}:\left(y_{1}, x_{2}, 0, \xi_{2}\right) \in \mathrm{WF}_{\mathrm{cl}}(u)\right\} .
\end{aligned}
$$

Proposition 2.8. Let $A \in \mathrm{L}_{\mathrm{cl}}^{m_{1}, m_{2}}\left(\Omega_{1}, \Omega_{2}\right)$ be bi-elliptic. Then

$$
\widetilde{\mathrm{WF}_{\mathrm{cl}}}(A u)=\widetilde{\mathrm{WF}_{\mathrm{cl}}}(u) \text {. }
$$


Proof. We already know that $\widetilde{\mathrm{WF}_{\mathrm{cl}}}(A u) \subset \widetilde{\mathrm{WF}_{\mathrm{cl}}}(u)$.

Now, $A \in \mathrm{L}_{\mathrm{cl}}^{m_{1}, m_{2}}\left(\Omega_{1}, \Omega_{2}\right)$, therefore there exists $B \in \mathrm{L}_{\mathrm{cl}}^{-m_{1},-m_{2}}\left(\Omega_{1}, \Omega_{2}\right)$ such that $B A-I=K \in \mathrm{L}^{-\infty,-\infty}\left(\Omega_{1}, \Omega_{2}\right)$. Thus

$$
\begin{aligned}
\widetilde{\mathrm{WF}_{\mathrm{cl}}}(u):=\widetilde{\mathrm{WF}_{\mathrm{cl}}}((B A-K) u) & \subset \widetilde{\mathrm{WF}_{\mathrm{cl}}}(B A u) \cup \widetilde{\mathrm{WF}_{\mathrm{cl}}}(K u) \\
& \subset \widetilde{\mathrm{WF}_{\mathrm{cl}}}(A u) \cup \emptyset=\widetilde{\mathrm{WF}_{\mathrm{cl}}}(A u) .
\end{aligned}
$$

This notion encourages to study microlocal properties of bisingular operators with respect to this modified notion of $\widetilde{\mathrm{WF}}(u)$. However, all of the previous results have been obtained by the study of distribution kernels. It is far more desirable to have a notion of singularities in terms of the actual calculus. This will be provided in the next section.

\section{A WAVE FRONT SET IN TERMS OF BISINGUlAR OPERATORS}

3.1. Microlocal properties of bisingular operators. While being the description that naturally arises when analysing the kernels of bisingular operators, the notion of wave front set used in the previous section has several drawbacks: it is defined in terms of the Hörmander wave front set, so in order to calculate it one first has to find that set and then "forget information". Also, it is not defined in terms of the bisingular calculus, but indeed with respect to the pseudodifferential one.

In the following we establish a second notion that does not have these drawbacks. In fact it turns out to be quite similar to the notion introduced in [CM03 for the $S G$-calculus. This is not surprising, as there is a strong similarity in the formulas arising in both calculi.

From now on, all the pseudodifferential operators will be assumed as properly supported, and all the bisingular operators to be classical.

Definition 3.1. Let $u \in \mathcal{D}^{\prime}\left(\Omega_{1} \times \Omega_{2}\right)$. We define $\mathrm{WF}_{\mathrm{bi}}(u) \subset \Omega_{1} \times \Omega_{2} \times$ $\left(\mathbb{R}^{n_{1}+n_{2}} \backslash 0\right)$ as

$$
\mathrm{WF}_{\mathrm{bi}}(u)=\mathrm{WF}_{\mathrm{bi}}^{1}(u) \cup \mathrm{WF}_{\mathrm{bi}}^{2}(u) \cup \mathrm{WF}_{\mathrm{bi}}^{12}(u)
$$

where

- $\left(x_{1}, x_{2}, \xi_{1}, 0\right)$ is not in $\mathrm{WF}_{\mathrm{bi}}^{1}(u)$ if there exists an $A \in \mathrm{L}_{\mathrm{cl}}^{0}\left(\Omega_{1}\right)$ noncharacteristic at $\left(x_{1}, \xi_{1}\right)$ such that

$$
(A \otimes I) u \in \mathcal{C}^{\infty}\left(\Omega_{1}, \mathcal{D}^{\prime}\left(\Omega_{2}\right)\right) .
$$

- $\left(x_{1}, x_{2}, 0, \xi_{2}\right)$ is not in $\mathrm{WF}_{\mathrm{bi}}^{2}(u)$ if there exists an $A \in \mathrm{L}_{\mathrm{cl}}^{0}\left(\Omega_{2}\right)$ noncharacteristic at $\left(x_{2}, \xi_{2}\right)$ such that

$$
(I \otimes A) u \in \mathcal{C}^{\infty}\left(\Omega_{2}, \mathcal{D}^{\prime}\left(\Omega_{1}\right)\right) .
$$

- $\left(x_{1}, x_{2}, \xi_{1}, \xi_{2}\right),\left|\xi_{1}\right|\left|\xi_{2}\right| \neq 0$, is not in $\mathrm{WF}_{\mathrm{bi}}^{12}(u)$ if there exists a $A_{i} \in$ $\mathrm{L}_{\mathrm{cl}}^{0}\left(\Omega_{i}\right)$, non-characteristic at $\left(x_{i}, \xi_{i}\right), i=1,2$, such that

$$
\begin{aligned}
\left(A_{1} \otimes A_{2}\right) u & \in \mathcal{C}^{\infty}\left(\Omega_{1} \times \Omega_{2}\right) \\
\left(A_{1} \otimes I\right) u & \in \mathcal{C}^{\infty}\left(\Omega_{1}, \mathcal{D}^{\prime}\left(\Omega_{2}\right)\right), \\
\left(I \otimes A_{2}\right) u & \in \mathcal{C}^{\infty}\left(\Omega_{2}, \mathcal{D}^{\prime}\left(\Omega_{1}\right)\right) .
\end{aligned}
$$


Remark 3.2. Note that the conditions (7) and (8) do not follow from (6): Take $u \in \mathcal{D}^{\prime}(\mathbb{R} \times \mathbb{R}), u=\delta(x-1) \delta(y+1)$ and $\psi$ smooth such that $\psi \equiv 1$ for $x>1 / 2$ and $\psi \equiv 0$ for $x \leq 0$. Then $(\psi(x) \otimes \psi(y)) u=0$, as $(1,-1) \notin \operatorname{supp}(\psi \otimes \psi)$. However, for $g \in \mathcal{D}(\mathbb{R})$ with $g(-1) \neq 0$ we have $(\psi(x) \otimes I) u(. \otimes g)=\delta(x-1) g(-1)$, which is not smooth.

Remark 3.3. For a distribution of the form $u=u_{1} \otimes u_{2}$, we have that $\mathrm{WF}_{\mathrm{bi}}^{12}(u)=\mathrm{WF}_{\mathrm{cl}}\left(u_{1}\right) \times \mathrm{WF}_{\mathrm{cl}}\left(u_{2}\right)$.

In fact we have the following inclusion result:

Lemma 3.3.1. If a point $\left(x_{1}, x_{2}, \xi_{1}, \xi_{2}\right),\left|\xi_{1}\right|\left|\xi_{2}\right| \neq 0$, is not in $\mathrm{WF}_{\mathrm{bi}}^{12}(u)$, then it is not in $\mathrm{WF}_{\mathrm{cl}}(u)$.

Proof. The proof is a variant of [Hör91], Proposition 2.8. By definition there exists $A:=A_{1} \otimes A_{2} \in \mathrm{L}^{0,0}\left(\Omega_{1} \times \Omega_{2}\right)$, with $\sigma^{12}(A)\left(x_{1}, x_{2}, \xi_{1}, \xi_{2}\right) \neq 0$, such that $A u \in \mathcal{C}^{\infty}\left(\Omega_{1} \times \Omega_{2}\right)$. Now take a ( $\Psi$ DO symbol) $\psi\left(x_{1}, x_{2}, \xi_{1}, \xi_{2}\right) \in$ $\mathrm{L}_{\mathrm{cl}}^{0}\left(\Omega_{1} \times \Omega_{2}\right)$ such that

- on the support of $\psi$ we have $\left\langle\xi_{1}\right\rangle \lesssim\left\langle\xi_{2}\right\rangle \lesssim\left\langle\xi_{1}\right\rangle$

- $\psi$ is non-characteristic at $\left(x_{1}, x_{2}, \xi_{1}, \xi_{2}\right)$.

Then the (bi-singular) operator product

$$
B:=\psi\left(x_{1}, x_{2}, D_{1}, D_{2}\right) \circ A\left(x_{1}, x_{2}, D_{1}, D_{2}\right)
$$

yields a pseudodifferential operator of order 0 , plus a smoothing remainder, by virtue of the above inequality on the support of $\psi$ and the symbol expand in Theorem 1.7. It has the following properties:

- its principal symbol is $\psi \cdot \sigma^{12}(A)$, and thus is non-characteristic (in the sense of $\Psi$ DOs) at $\left(x_{1}, x_{2}, \xi_{1}, \xi_{2}\right)$ and of zero order.

- $B u=\psi\left(x_{1}, x_{2}, D_{1}, D_{2}\right) A\left(x_{1}, x_{2}, D_{1}, D_{2}\right) u \in \mathcal{C}^{\infty}$

This proves the claim.

Remark 3.4. This lemma asserts that in the conic region where both covariables are non-vanishing we can pass from bisingular to pseudodifferential calculus by multiplying by a $\Psi D O$. This has the consequence that the two operator classes have similar microlocal properties (with respect to the classical wave front set) in that region.

The following Lemma gives a similar interpretation to the remaining components, illustrating the loss of localization of singularities already encountered in the previous section.

Lemma 3.4.1. Let $u \in \mathcal{E}^{\prime}\left(\Omega_{1} \times \Omega_{2}\right),\left(x_{1}^{0}, \xi_{1}^{0}\right) \in \Omega_{1} \times\left(\mathbb{R}^{n_{1}} \backslash 0\right)$. If for all $y \in \Omega_{2}$ we have $\left(x_{1}^{0}, y, \xi_{1}^{0}, 0\right) \notin \mathrm{WF}_{\mathrm{cl}}(u)$ then for all $y \in \Omega_{2}$ we have $\left(x_{1}^{0}, y, \xi_{1}^{0}, 0\right) \notin \mathrm{WF}_{\mathrm{bi}}^{1}(u)$.

Similarly, if for all $x \in \Omega_{1}$ we have $\left(x, x_{2}^{0}, 0, \xi_{2}^{0}\right) \notin \mathrm{WF}_{\mathrm{cl}}(u)$ then for all $x \in \Omega_{1}$ we have $\left(x, x_{2}^{0}, 0, \xi_{2}^{0}\right) \notin \mathrm{WF}_{\mathrm{bi}}^{2}(u)$.

Proof. We prove the claim for $\mathrm{WF}_{\mathrm{bi}}^{1}$. Take $\left(x_{1}^{0}, y, \xi_{1}^{0}, 0\right) \notin \mathrm{WF}_{\mathrm{cl}}(u)$. Then there exist a cut-off $\phi \in \mathcal{C}_{0}^{\infty}\left(\Omega_{1} \times \Omega_{2}\right)$ and a conic localizer $\psi_{y} \in \mathcal{C}^{\infty}\left(\mathbb{R}^{n_{1}+n_{2}}\right)$, non-vanishing in a (conic) neighbourhood $\left(x_{1}^{0}, y\right)$ and $\left(\xi_{1}^{0}, 0\right)$ respectively, such that

$$
\psi_{y}\left(\xi_{1}, \xi_{2}\right) \mathcal{F}_{\left(x_{1}, x_{2}\right) \mapsto\left(\xi_{1}, \xi_{2}\right)}\left\{\phi\left(x_{1}, x_{2}\right) u\right\} \in \mathscr{S}\left(\mathbb{R}^{n_{1}+n_{2}}\right) .
$$


As this holds true for any $y$, and due to compactness of the support of $u$, there exists a cut-off $\phi_{1} \in \mathcal{C}_{0}^{\infty}\left(\Omega_{1}\right)$ such that for some conic localizer $\psi \in \mathcal{C}^{\infty}\left(\mathbb{R}^{n_{1}+n_{2}}\right)$

$$
\psi\left(\xi_{1}, \xi_{2}\right) \mathcal{F}_{\left(x_{1}, x_{2}\right) \mapsto\left(\xi_{1}, \xi_{2}\right)}\left\{\phi\left(x_{1}\right) u\right\} \in \mathscr{S}\left(\mathbb{R}^{n_{1}+n_{2}}\right) .
$$

This means we can find a conic localizer $\psi_{1} \in \mathcal{C}^{\infty}\left(\mathbb{R}^{n_{1}}\right)$, non-vanishing around $\xi_{1}^{0}$, such that $\psi\left(\xi_{1}\right) \mathcal{F}_{\left(x_{1}, x_{2}\right) \mapsto\left(\xi_{1}, \xi_{2}\right)}\left\{\phi\left(x_{1}\right) u\right\} \in \mathcal{C}^{\infty}\left(\mathbb{R}^{n_{1}+n_{2}}\right)$, rapidly decaying with respect to the first variable $\xi_{1}$ for fixed $\xi_{2}$, and polynomially bounded everywhere, by the Paley-Wiener-Schwartz Theorem.

We define $A \in \mathrm{L}_{\mathrm{cl}}^{0}\left(\Omega_{1}\right)$ as the operator

$$
A v\left(y_{1}\right)=\mathcal{F}_{\xi_{1} \mapsto y_{1}}^{-1}\left(\psi\left(\xi_{1}\right) \mathcal{F}_{x_{1} \mapsto \xi_{1}}\left\{\phi\left(x_{1}\right) u\right\} .\right.
$$

By the assumptions on $\phi_{1}$ and $\psi_{1}, A$ is non-characteristic in the sense of pseudodifferential operators at $\left(x_{1}^{0}, \xi_{1}^{0}\right)$. But for any $f \in \mathcal{D}\left(\Omega_{2}\right)$ we have that $[(A \otimes I) u](f)\left(x_{1}\right)=\mathcal{F}_{\xi_{1} \mapsto x_{1}}^{-1}\left\langle\psi\left(\xi_{1}\right) \mathcal{F}_{\left(y_{1}, y_{2}\right) \mapsto\left(\xi_{1}, \xi_{2}\right)}\left\{\phi\left(y_{1}\right) u\right\}, \widehat{f}\right\rangle$ is a smooth function, which means $(A \otimes I) u \in \mathcal{C}^{\infty}\left(\Omega_{1}, \mathcal{D}^{\prime}\left(\Omega_{2}\right)\right)$.

Proposition 3.5. Let $u \in \mathcal{E}^{\prime}\left(\Omega_{1} \times \Omega_{2}\right)$. Then

$$
\mathrm{WF}_{\mathrm{bi}}(u)=\emptyset \Leftrightarrow u \in \mathcal{C}^{\infty}\left(\Omega_{1} \times \Omega_{2}\right) .
$$

Proof. Assume $\mathrm{WF}_{\mathrm{bi}}(u)=\emptyset$. Then, by virtue of Lemma 3.3.1, we have $\mathrm{WF}_{\mathrm{cl}}(u) \cap\left\{\left(x_{1}, x_{2}, \xi_{1}, \xi_{2}\right)|:| \xi_{1}|| \xi_{2} \mid \neq 0\right\}=\emptyset$. Thus $\widehat{u}$ is rapidly decaying on any ray $\mathbb{R} \cdot\left(\xi_{1}, \xi_{2}\right)$ where $\left|\xi_{1}\right|\left|\xi_{2}\right| \neq 0$.

As also $\mathrm{WF}_{\mathrm{bi}}^{1}(u)=\emptyset$ we can find for each $\left(x_{1}, x_{2}, \xi_{1}, \xi_{2}\right)$ an $A \in \mathrm{L}_{\mathrm{cl}}^{0}\left(\Omega_{1}\right)$ noncharacteristic at $\left(x_{1}, \xi_{1}\right)$ such that, by Lemma 2.4.1, for any $B \in \mathrm{L}^{m,-\infty}\left(\Omega_{1} \times\right.$ $\left.\Omega_{2}\right)$ we have $B(I \otimes A) u \in \mathcal{C}^{\infty}\left(\Omega_{1} \times \Omega_{2}\right)$. By compactness and a parametrix construction we can thus conclude that for all $B \in \mathrm{L}^{m,-\infty}\left(\Omega_{1} \times \Omega_{2}\right)$ we get $B u \in \mathcal{C}^{\infty}\left(\Omega_{1} \times \Omega_{2}\right)$. Now pick $\phi \in \mathcal{C}_{0}^{\infty}\left(\Omega_{1} \times \Omega_{2}\right)$ with $\phi \equiv 1$ on the support of $u$, and define $b\left(x_{1}, x_{2}, \xi_{1}, \xi_{2}\right)=\phi\left(x_{1}, x_{2}\right) f\left(\xi_{2}\right)$, with $f \in \mathscr{S}\left(\mathbb{R}^{d_{2}}\right)$. Then

$$
\mathscr{S}\left(\mathbb{R}^{d_{1}+d_{2}}\right) \ni \mathcal{F}(B u)=\mathcal{F}\left(b\left(x_{1}, x_{2}, D_{1}, D_{2}\right) u\right)=(1 \otimes f) \widehat{u} .
$$

As $f$ was arbitrary and rapidly decaying, this means that $\widehat{u}$ must already be rapidly decaying in the first variable. Repeating the argument for the second variable proves the assertion.

Using a parametrix construction, we get by the same arguments:

Proposition 3.6. Let $u \in \mathcal{E}^{\prime}\left(\Omega_{1} \times \Omega_{2}\right)$. Then

$$
\begin{aligned}
& \mathrm{WF}_{\mathrm{bi}}^{1}(u)=\emptyset \Leftrightarrow u \in \mathcal{C}^{\infty}\left(\Omega_{1}, \mathcal{D}^{\prime}\left(\Omega_{2}\right)\right), \\
& \mathrm{WF}_{\mathrm{bi}}^{2}(u)=\emptyset \Leftrightarrow u \in \mathcal{C}^{\infty}\left(\Omega_{2}, \mathcal{D}^{\prime}\left(\Omega_{1}\right)\right) .
\end{aligned}
$$

Remark 3.7. Note that $u \in \mathcal{C}^{\infty}\left(\Omega_{1}, \mathcal{D}^{\prime}\left(\Omega_{2}\right)\right) \cap \mathcal{C}^{\infty}\left(\Omega_{2}, \mathcal{D}^{\prime}\left(\Omega_{1}\right)\right)$ does not imply that $u \in \mathcal{C}^{\infty}\left(\Omega_{1} \times \Omega_{2}\right)$. Following [Trè67, a counterexample is, for instance, $\delta\left(x_{1}-x_{2}\right)$. The additional regularity needed such that $u \in \mathcal{C}^{\infty}\left(\Omega_{1} \times\right.$ $\left.\Omega_{2}\right)$ is therefore, by Proposition 3.5, encoded in $\mathrm{WF}_{\mathrm{bi}}^{12}(u)$.

The bisingular wave front set admits the following properties:

Proposition 3.8 (Properties of $\left.\mathrm{WF}_{\mathrm{bi}}\right)$. Let $u, v \in \mathcal{D}^{\prime}\left(\Omega_{1} \times \Omega_{2}\right), f \in$ $\mathcal{C}^{\infty}\left(\Omega_{1} \times \Omega_{2}\right)$. 
- $\mathrm{WF}_{\mathrm{bi}}$ a closed set and is conic with respect to both covariables jointly.

- Let $\Omega_{1}=\Omega_{2}=\Omega$. Define for $f \in \mathcal{C}^{\infty}(\Omega \times \Omega) A u\left(x_{1}, x_{2}\right)=u\left(x_{2}, x_{1}\right)$. Then we can define the pull-back $A^{*}$ as an endomorphism on $\mathcal{D}^{\prime}(\Omega \times \Omega)$ by duality and we have $\left(x_{1}, x_{2}, \xi_{1}, \xi_{2}\right) \in \mathrm{WF}_{\mathrm{bi}}(A u) \Leftrightarrow$ $\left(x_{2}, x_{1}, \xi_{2}, \xi_{1}\right) \in \mathrm{WF}_{\mathrm{bi}}(u)$.

- $\mathrm{WF}_{\mathrm{bi}}(u+v) \subset \mathrm{WF}_{\mathrm{bi}}(u) \cup \mathrm{WF}_{\mathrm{bi}}(v) ; \mathrm{WF}_{\mathrm{bi}}(f u) \subset \mathrm{WF}_{\mathrm{bi}}(u)$.

Remark 3.9. These properties are quite similar to the ones the $S G$-wave front set of [CM03 admits. This is not very surprising, as the bisingular calculus is formally very similar in its definition to the $S G$-calculus, through which the $S G$-wave front set is introduced. We will explore this connection in Section 4 .

Lemma 3.9.1. Let $C \in \mathrm{L}^{m_{1}, m_{2}}\left(\Omega_{1} \times \Omega_{2}\right), u \in \mathcal{D}^{\prime}\left(\Omega_{1} \times \Omega_{2}\right)$. Then we have $\mathrm{WF}_{\mathrm{bi}}^{1}(C u) \subset \mathrm{WF}_{\mathrm{bi}}^{1}(u)$.

Proof. Let $\left(x_{1}, x_{2}, \xi_{1}, 0\right) \notin \mathrm{WF}_{\mathrm{bi}}^{1}(u)$. By definition there exists a non-char (at $\left.\left(x_{1}, \xi_{1}\right)\right) A \in \mathrm{L}_{\mathrm{cl}}^{0}\left(\Omega_{1}\right)$ such that $(A \otimes I) u \in \mathcal{C}^{\infty}\left(\Omega_{1}, \mathcal{D}^{\prime}\left(\Omega_{2}\right)\right.$. In particular, by Lemma 2.4.1 we have $\forall B \in \mathrm{L}^{m,-\infty}\left(\Omega_{1} \times \Omega_{2}\right)$ that $B(A \otimes I) u$ is smooth. By the standard pseudodifferential calculus we can thus find an $A^{\prime} \in \mathrm{L}^{0}\left(\Omega_{1}\right)$ such that $A+A^{\prime}$ is elliptic in the sense of pseudodifferential operators and such that the symbol of $A^{\prime}$ vanishes on a conic neighbourhood $\Gamma$ of $\left(x_{1}, \xi_{1}\right)$. We thus have a parametrix $P \in \mathrm{L}^{0}\left(\Omega_{1}\right)$ such that $P\left(A+A^{\prime}\right)=I-R$ with $R \in \mathrm{L}^{-\infty}\left(\Omega_{1}\right)$.

Take $H \in \mathrm{L}^{0}\left(\Omega_{1}\right)$ such that $H$ is non-characteristic at $\left(x_{1}, \xi_{1}\right)$ and such that the symbol of $H$ vanishes outside a proper subcone of $\Gamma$. Then we have:

$$
\begin{aligned}
(H \otimes I) C u & =(H \otimes I) C\left(\left(P\left(A+A^{\prime}\right)+R\right) \otimes I\right) u \\
& =(H \otimes I) C(P \otimes I)(A \otimes I) u+(H \otimes I) C\left(P A^{\prime} \otimes I\right) u+ \\
& +(H \otimes I) C(R \otimes I) u \in \mathcal{C}^{\infty}\left(\Omega_{1}, \mathcal{D}^{\prime}\left(\Omega_{2}\right)\right) .
\end{aligned}
$$

The first summand is in $\mathcal{C}^{\infty}\left(\Omega_{1}, \mathcal{D}^{\prime}\left(\Omega_{2}\right)\right)$ due to the definition of $A$, the second as the symbol expansion given in Theorem 1.7, using the support properties of the symbols of $H$ and $A^{\prime}$, gives an operator in $L^{-\infty, 0}$. The third one is in $\mathcal{C}^{\infty}\left(\Omega_{1}, \mathcal{D}^{\prime}\left(\Omega_{2}\right)\right)$ as $R \in \mathrm{L}^{-\infty, 0}$ is already a smoothing operator in the first variable. This proves the claim.

Lemma 3.9.2. Let $C \in \mathrm{L}^{m_{1}, m_{2}}\left(\Omega_{1} \times \Omega_{2}\right), u \in \mathcal{D}^{\prime}\left(\Omega_{1} \times \Omega_{2}\right)$. Then we have $\mathrm{WF}_{\mathrm{bi}}^{12}(C u) \subset \mathrm{WF}_{\mathrm{bi}}^{12}(u)$.

Proof. Let $\left(x_{1}, x_{2}, \xi_{1}, \xi_{2}\right) \notin \mathrm{WF}_{\mathrm{bi}}^{12}(u)$. Then, by definition, we know there exist $A_{i} \in \mathrm{L}_{\mathrm{cl}}^{0}\left(\Omega_{i}\right)$, non-char at $\left(x_{i}, \xi_{i}\right), i=1,2$, such that

$$
\begin{aligned}
\left(A_{1} \otimes A_{2}\right) u & \in \mathcal{C}^{\infty}\left(\Omega_{1} \times \Omega_{2}\right), \\
\left(A_{1} \otimes I\right) u & \in \mathcal{C}^{\infty}\left(\Omega_{1}, \mathcal{D}^{\prime}\left(\Omega_{2}\right)\right), \\
\left(I \otimes A_{2}\right) u & \in \mathcal{C}^{\infty}\left(\Omega_{2}, \mathcal{D}^{\prime}\left(\Omega_{1}\right)\right) .
\end{aligned}
$$

By the standard pseudodifferential calculus we can thus find $A_{i}^{\prime} \in \mathrm{L}^{0}\left(\Omega_{i}\right)$ such that $A_{i}+A_{i}^{\prime}$ is elliptic in the sense of pseudodifferential operators and such that the symbol of $A_{i}^{\prime}$ vanishes on a conic neighborhood $\Gamma_{i}$ of $\left(x_{i}, \xi_{i}\right)$. 
We then have two parametrices $P_{i} \in \mathrm{L}^{0}\left(\Omega_{i}\right)$ such that

$$
\left(P_{1} \otimes P_{2}\right)\left(\left(A_{1}+A_{1}^{\prime}\right) \otimes\left(A_{2}+A_{2}^{\prime}\right)\right)=I \otimes I-R_{1} \otimes I-I \otimes R_{2}-\underbrace{R_{1} \otimes R_{2}}_{:=R},
$$

with $R_{i} \in \mathrm{L}^{-\infty}\left(\Omega_{i}\right)$. Now pick $H_{i} \in \mathrm{L}^{0}\left(\Omega_{i}\right)$ such that $H_{i}$ is non-characteristic at $\left(x_{i}, \xi_{i}\right)$ and such that the symbol of $H_{i}$ vanishes outside a proper subcone of $\Gamma_{i}$. Then, recalling that by the standard pseudodifferential calculus if two operators have disjoint support their product is a smoothing operator, and using Lemma 2.4.1.

$$
\begin{aligned}
& \left(H_{1} \otimes H_{2}\right) C u= \\
& \left(H_{1} \otimes H_{2}\right) C\left(\left(P_{1} \otimes P_{2}\right)\left(\left(A_{1}+A_{1}^{\prime}\right) \otimes\left(A_{2}+A_{2}^{\prime}\right)\right)+R_{1} \otimes I+I \otimes R_{2}+R\right) u \\
& =\underbrace{\left(H_{1} \otimes H_{2}\right) C\left(P_{1} \otimes P_{2}\right)\left(A_{1} \otimes A_{2}\right) u}_{\in \mathcal{C}^{\infty} \text { by eq (6) }}+\underbrace{\left(H_{1} \otimes H_{2}\right) C\left(P_{1} \otimes P_{2}\right)\left(A_{1}^{\prime} \otimes A_{2}\right) u}_{\in \mathcal{C}^{\infty} \text { by (8) and by the support of } H_{1}, A_{1}^{\prime}} \\
& +\underbrace{\left(H_{1} \otimes H_{2}\right) C\left(P_{1} \otimes P_{2}\right)\left(A_{1} \otimes A_{2}^{\prime}\right) u}_{\in \mathcal{C}^{\infty} \text { by (7) and by the support of } H_{2}, A_{2}^{\prime}}+\underbrace{\left(H_{1} \otimes H_{2}\right) C\left(P_{1} \otimes P_{2}\right)\left(A_{1}^{\prime} \otimes A_{2}^{\prime}\right) u}_{\in \mathcal{C}^{\infty} \text { by the support of } H_{1}, H_{2}, A_{1}^{\prime}, A_{2}^{\prime}} \\
& +\left(H_{1} \otimes H_{2}\right) C\left(R_{1} \otimes I\right) u+\left(H_{1} \otimes H_{2}\right) C\left(I \otimes R_{2}\right) u+\underbrace{\left(H_{1} \otimes H_{2}\right) C R u}_{\in \mathcal{C}^{\infty} \text { because } R \in \mathrm{L}^{-\infty,-\infty}} \\
& =\left(H_{1} \otimes H_{2}\right) C\left(R_{1} \otimes I\right) u+\left(H_{1} \otimes H_{2}\right) C\left(I \otimes R_{2}\right) u \quad \bmod \mathcal{C}^{\infty} .
\end{aligned}
$$

Now, without loss of generality, we proceed with the calculations only for the term $\left(H_{1} \otimes H_{2}\right) C\left(R_{1} \otimes I\right) u$. We have

$$
\begin{aligned}
& \left(H_{1} \otimes H_{2}\right) C\left(R_{1} \otimes I\right) u=\left(H_{1} \otimes H_{2}\right) C\left(R_{1} \otimes\left(P_{2}\left(A_{2}+A_{2}^{\prime}\right)+R_{2}\right)\right) u \\
& =\underbrace{\left(H_{1} \otimes H_{2}\right) C\left(R_{1} \otimes P_{2}\right)\left(I \otimes A_{2}\right) u}_{\left.\in \mathcal{C}^{\infty} \text { by } \mathbb{8}\right)} \\
& +\underbrace{\left(H_{1} \otimes H_{2}\right) C\left(R_{1} \otimes P_{2}\right)\left(I \otimes A_{2}^{\prime}\right) u}_{\in \mathcal{C}^{\infty} \text { by the support of } H_{2}, A_{2}^{\prime}} \\
& +\underbrace{\left(H_{1} \otimes H_{2}\right) C\left(R_{1} \otimes I\right)\left(I \otimes R_{2}\right) u}_{\in \mathcal{C}^{\infty} \text { because } R \in \mathrm{L}^{-\infty,-\infty}} \in \mathcal{C}^{\infty},
\end{aligned}
$$

therefore $\left(H_{1} \otimes H_{2}\right) C u \in \mathcal{C}^{\infty}$. With similar computations, one can check that

$$
\begin{aligned}
& \left(H_{1} \otimes I\right) C u \in \mathcal{C}^{\infty}\left(\Omega_{1}, \mathcal{D}^{\prime}\left(\Omega_{2}\right)\right) \\
& \left(I \otimes H_{2}\right) C u \in \mathcal{C}^{\infty}\left(\Omega_{2}, \mathcal{D}^{\prime}\left(\Omega_{1}\right)\right)
\end{aligned}
$$

and this proves the claim.

The preceding Lemmas lead to the following proposition, which asserts that this definition of wave front set is indeed suitable for the calculus of bisingular operators:

Proposition 3.10 (Microlocality of bisingular operators). Let $C \in$ $\mathrm{L}^{m_{1}, m_{2}}\left(\Omega_{1} \times \Omega_{2}\right), u \in \mathcal{D}^{\prime}\left(\Omega_{1} \times \Omega_{2}\right)$. Then we have $\mathrm{WF}_{\mathrm{bi}}(C u) \subset \mathrm{WF}_{\mathrm{bi}}(u)$. 
3.2. Microelliptic properties of bisingular operators. From the previous proposition, we can conclude that bielliptic operators preserve the bi-wave front set:

Corollary 3.10.1. Let $A \in \mathrm{L}^{m_{1}, m_{2}}\left(\Omega_{1}, \Omega_{2}\right)$ be bi-elliptic. Then $\mathrm{WF}_{\mathrm{bi}}(A u)=\mathrm{WF}_{\mathrm{bi}}(u)$.

Proof. One inclusion follows directly form Proposition 3.10. The other follows proceeding like in Proposition 2.8

Next we study the microellipticity properties of bisingular operators. For that we need a suitable definition of a characteristic set. As in Definition 1.4, $\Omega_{1}$ and $\Omega_{2}$ are considered as compact manifolds.

Definition 3.11. Let $B \in \mathrm{L}^{m_{1}, m_{2}}\left(\Omega_{1}, \Omega_{2}\right)$ and $v_{0}=\left(x_{1}, \xi_{1}\right) \in \Omega_{1} \times\left(\mathbb{R}^{n_{1}} \backslash 0\right)$. We say that $B$ is not 1-characteristic at $V:=\pi_{2}^{-1} v_{0}:=\left\{\left(x_{1}, y, \xi_{1}, 0\right): y \in\right.$ $\left.\Omega_{2}\right\}$ if

(1) for all $v \in V$ there exists an open conic neighbourhood $\Theta$ of $v$ such that $\sigma^{12} \neq 0$ on $\Theta \backslash\left(\mathbb{R}^{+} v\right)$,

(2) $\sigma^{1}(B) \in \mathrm{L}_{\mathrm{cl}}^{m_{2}}\left(\Omega_{2}\right)$ is invertible with inverse in $\mathrm{L}_{\mathrm{cl}}^{-m_{2}}\left(\Omega_{2}\right)$ in an open conic neighbourhood $\Gamma$ of $v_{0}$.

Let $\operatorname{Char}_{\mathrm{bi}}^{1}(B)$ be the set of all $V$ such that $B$ is 1 -characteristic at $V$.

We define $\operatorname{Char}_{\mathrm{bi}}^{2}(B)$ accordingly for $W:=\pi_{2}^{-1} w_{0}, w_{0} \in \Omega_{2} \times\left(\mathbb{R}^{n_{2}} \backslash 0\right)$, by exchanging the roles of $\sigma^{1}(B)$ and $\sigma^{2}(B)$.

Finally, we define $\operatorname{Char}_{\mathrm{bi}}^{12}(B)$ as the set of points $z=\left(x_{1}, x_{2}, \xi_{1}, \xi_{2}\right),\left|\xi_{1}\right|\left|\xi_{2}\right| \neq$ 0 , where $\sigma^{12}(z)=0$.

Set

$$
\operatorname{Char}_{\mathrm{bi}}(B):=\operatorname{Char}_{\mathrm{bi}}^{1}(B) \cup \operatorname{Char}_{\mathrm{bi}}^{2}(B) \cup \operatorname{Char}_{\mathrm{bi}}^{12}(B) .
$$

Remark 3.12. $B$ is bi-elliptic iff $\operatorname{Char}_{\mathrm{bi}}(B)=\emptyset$.

Remark 3.13. With this notion Definition 3.1 can also be expressed in the form

$$
\begin{aligned}
& \mathrm{WF}_{\mathrm{bi}}^{1}(u)=\bigcap_{\substack{A \in \mathrm{L}_{\mathrm{cl}}^{0}\left(\Omega_{1}\right) \\
(A \otimes I) u \in \mathcal{C}^{\infty}\left(\Omega_{1}, \mathcal{D}^{\prime}\left(\Omega_{2}\right)\right)}} \underbrace{\operatorname{Char}_{\mathrm{bi}}^{1}(A \otimes I)}_{\operatorname{Char}_{\mathrm{cl}}(A) \times\left(\Omega_{2} \times\left(\mathbb{R}^{n_{2}} \backslash 0\right)\right)} \\
& \mathrm{WF}_{\mathrm{bi}}^{2}(u)=\bigcap_{\substack{A \in \mathrm{L}_{\mathrm{cl}}^{0}\left(\Omega_{2}\right) \\
(I \otimes A) u \in \mathcal{C}^{\infty}\left(\Omega_{2}, \mathcal{D}^{\prime}\left(\Omega_{1}\right)\right)}} \underbrace{\operatorname{Char}_{\mathrm{bi}}^{2}(I \otimes A),}_{\left(\Omega_{1} \times\left(\mathbb{R}^{\left.\left.n_{1} \backslash 0\right)\right) \times \operatorname{Char}_{\mathrm{cl}}(A)}\right.\right.} \\
& \mathrm{WF}_{\mathrm{bi}}^{12}(u)=\bigcap_{A_{i} \in \mathrm{L}_{\mathrm{cl}}^{0}\left(\Omega_{1}\right)} \operatorname{Char}_{\mathrm{bi}}^{12}\left(A_{1} \otimes A_{2}\right) \text {. } \\
& \left(A_{1} \otimes A_{2}\right) u \in \mathcal{C}^{\infty} \\
& \left(A_{1} \otimes I\right) u \in \mathcal{C}^{\infty}\left(\Omega_{1}, \mathcal{D}^{\prime}\left(\Omega_{2}\right)\right) \\
& \left(I \otimes A_{2}\right) u \in \mathcal{C}^{\infty}\left(\Omega_{2}, \mathcal{D}^{\prime}\left(\Omega_{1}\right)\right)
\end{aligned}
$$

With the definition of $\mathrm{Char}_{\mathrm{bi}}$ we can review in the following Table 3.2 the model cases of Table 1.1 , setting $\mathbb{R}_{0}^{n}=\mathbb{R}^{n} \backslash 0, \Omega=\Omega_{1} \times \Omega_{2}$ and $\mathbb{R}_{0}^{n_{12}}=$ $\mathbb{R}_{0}^{n_{1}} \times \mathbb{R}_{0}^{n_{2}}$.

Lemma 3.13.1. Let $C \in \mathrm{L}^{m_{1}, m_{2}}\left(\Omega_{1}, \Omega_{2}\right)$ be such that

$$
\operatorname{Char}_{\mathrm{bi}}^{1}(C) \cap\left(\Gamma \times \Omega_{2} \times\{0\}\right)=\emptyset .
$$




\begin{tabular}{|c|c|c|c|}
\hline Operator & Char $_{\mathrm{bi}}^{1}$ & Char $_{\mathrm{bi}}^{2}$ & Char $_{\mathrm{bi}}^{12}$ \\
\hline$I \otimes I$ & $\emptyset$ & $\emptyset$ & $\emptyset$ \\
\hline$-\Delta_{1} \otimes I+I \otimes\left(-\Delta_{2}\right)$ & $\Omega \times \mathbb{R}_{0}^{n_{1}} \times\{0\}$ & $\Omega \times\{0\} \times \mathbb{R}_{0}^{n_{2}}$ & $\Omega \times \mathbb{R}_{0}^{n_{12}}$ \\
\hline$-\Delta_{1} \otimes\left(-\Delta_{2}\right)$ & $\Omega \times \mathbb{R}_{0}^{n_{1}} \times\{0\}$ & $\Omega \times\{0\} \times \mathbb{R}_{0}^{n_{2}}$ & $\emptyset$ \\
\hline$-\Delta_{1} \otimes\left(-\Delta_{2}+I\right)$ & $\Omega \times \mathbb{R}_{0}^{n_{1}} \times\{0\}$ & $\emptyset$ & $\emptyset$ \\
\hline$\left(-\Delta_{1}+I\right) \otimes\left(-\Delta_{2}+I\right)$ & $\emptyset$ & $\emptyset$ & $\emptyset$ \\
\hline$\left(-\Delta_{1}+I\right)^{-1} \otimes\left(-\Delta_{2}+I\right)^{-1}$ & $\emptyset$ & $\emptyset$ & $\emptyset$ \\
\hline
\end{tabular}

TABLE 2. Char ${ }_{\mathrm{bi}}$ for model cases of bisingular operators

Let $a \in \mathrm{S}^{0}\left(\Omega_{1}\right)$ have support in a closed cone $\Gamma$ and be non-char (in the sense of $\Psi D O)$ in $\Gamma^{0}$. Then there exists a $H \in \mathrm{L}^{-m_{1},-m_{2}}\left(\Omega_{1}, \Omega_{2}\right)$ such that

$$
H C=A \otimes I-R,
$$

where $R \in \mathrm{L}^{-\infty, 0}$.

Proof. The requirements on the support of $a$ mean precisely that $C$ is elliptic with respect to $a$ in the sense of [Cor95, Theorem 2.3.3. Therefore, by the classical calculus of pseudodifferential operators, we can find a symbol $e \in \mathrm{S}^{-m_{1},-m_{2}}$ such that for all fixed $\left(x_{2}, \xi_{2}\right)$ the operator $E\left(x_{2}, \xi_{2}\right)=$ $e\left(x_{1}, x_{2}, D_{1}, \xi_{2}\right)$ is a local parametrix with respect to $a$ namely,

$$
E\left(x_{2}, \xi_{2}\right) \sigma^{2}(C)\left(x_{2}, \xi_{2}\right)=R\left(x_{2}, \xi_{2}\right)+(A \otimes 1)\left(x_{2}, \xi_{2}\right),
$$

where $R\left(x_{2}, D_{2}\right) \in \mathrm{L}^{-\infty, 0}\left(\Omega_{1}, \Omega_{2}\right)$ and $E\left(x_{2}, D_{2}\right) \in \mathrm{L}^{-m_{1},-m_{2}}\left(\Omega_{1}, \Omega_{2}\right)$.

Now define $H$ as the operator with principal symbol

$$
\begin{aligned}
h=\psi_{1}\left(x_{1}, \xi_{1}\right) a_{m_{1} ;} \cdot e_{m_{1} ; \cdot} & +\psi_{2}\left(x_{2}, \xi_{2}\right) a_{\cdot ; m_{2}} c_{* ; m_{2}}^{-1} \\
& -\psi_{1}\left(x_{1}, \xi_{1}\right) \psi_{2}\left(x_{2}, \xi_{2}\right) a_{m_{1} ; m_{2}} c_{m_{1} ; m_{2}}^{-1} .
\end{aligned}
$$

Using the calculus and Theorem 1.7, it is straightforward that $H$ matches the requirements.

Lemma 3.13.2. Let $C \in \mathrm{L}^{m_{1}, m_{2}}\left(\Omega_{1} \times \Omega_{2}\right), u \in \mathcal{D}^{\prime}\left(\Omega_{1} \times \Omega_{2}\right)$. Then we have

$$
\mathrm{WF}_{\mathrm{bi}}^{1}(u) \subseteq \operatorname{Char}_{\mathrm{bi}}^{1}(C) \cup \mathrm{WF}_{\mathrm{bi}}^{1}(C u) .
$$

Proof. Let $\left(x_{1}, x_{2}, \xi_{1}, 0\right) \notin \operatorname{Char}_{\mathrm{bi}}^{1}(C) \cup \mathrm{WF}_{\mathrm{bi}}^{1}(C u)$. Then there exists $A \in$ $\mathrm{L}^{0}\left(\Omega_{1}\right)$ such that we have $(A \otimes I) H C u \in \mathcal{C}^{\infty}\left(\Omega_{1}, \mathcal{D}^{\prime}\left(\Omega_{2}\right)\right)$, with $H$ as in Lemma 3.13.1, due to microlocality (Proposition 3.10) of $H$. Then we have

$$
\begin{aligned}
\left(A^{2} \otimes I\right) u & =(A \otimes I)(R-H C) u \\
& =\underbrace{(A \otimes I) R}_{\in \mathrm{L}^{-\infty, 0}} u-\underbrace{(A \otimes I) H C u}_{\in \mathcal{C}^{\infty}\left(\Omega_{1}, \mathcal{D}^{\prime}\left(\Omega_{2}\right)\right) \text { by assumption on } A} \in \mathcal{C}^{\infty} .
\end{aligned}
$$

Using Lemma 2.4.1 on the first summand, this proves the claim.

Using the previous Lemma, we conclude that

Proposition 3.14 (Microellipticity of bisingular operators with respect to the 1 and 2 components of $\left.\mathrm{WF}_{\mathrm{bi}}\right)$. Let $C \in \mathrm{L}^{m_{1}, m_{2}}\left(\Omega_{1}, \Omega_{2}\right)$, $u \in \mathcal{D}^{\prime}\left(\Omega_{1} \times \Omega_{2}\right)$. Then

$$
\mathrm{WF}_{\mathrm{bi}}^{i}(u) \subseteq \operatorname{Char}_{\mathrm{bi}}^{i}(C) \cup \mathrm{WF}_{\mathrm{bi}}^{i}(C u),
$$


$i=1,2$.

We do, however, not obtain full microellipticity, i.e. with respect to the $\mathrm{WF}_{\mathrm{bi}}^{12}$-component. This can be seen by the following example:

Example 3.15. Consider $C=-\Delta \otimes(-\Delta), u=\delta \otimes 1+1 \otimes \delta$. Then $\operatorname{Char}_{\text {bi }}^{12}(C)=\emptyset$ and $C u=0 \in \mathcal{C}^{\infty}\left(\Omega_{1} \times \Omega_{2}\right)$, i.e. $\operatorname{WF}_{\text {bi }}^{12}(C u)=\emptyset$. But take any $A_{1}$ non-characteristic at $\left(0, \xi_{1}\right), \xi_{1} \neq 0$. Then $\left(A_{1} \otimes I\right) u=\left(A_{1} \delta\right) \otimes 1$ which is never in $\mathcal{C}^{\infty}\left(\Omega_{1}, \mathcal{D}^{\prime}\left(\Omega_{2}\right)\right)$, and this means that $\mathrm{WF}_{\mathrm{bi}}^{12}(u)$ is nonempty, because the (7)- and, by a similar argument, (8)-requirements fail to hold.

Remark 3.16. The counterexample to full microellipticity could be circumvented by imposing stronger invertibility conditions in the definition of $\mathrm{Char}_{\mathrm{bi}}^{12}$. This would, however, break the characterization of the wave front set of Remark 3.13, and lead to a loss of local information, while yielding no interesting cases not already covered by Corollary 3.10.1.

With our definition we obtain, however, the following Lemma, which can be regarded as a microellipticity result for the ([6)-part of Definition 3.1, for operators given by a tensor product.

Lemma 3.16.1. Let $C_{i} \in \mathrm{L}^{m_{i}}\left(\Omega_{i}\right), u \in \mathcal{D}^{\prime}\left(\Omega_{1} \times \Omega_{2}\right)$. If

$$
\left(x_{1}, x_{2}, \xi_{1}, \xi_{2}\right) \notin \operatorname{Char}_{\mathrm{bi}}^{12}\left(C_{1} \otimes C_{2}\right) \cup \mathrm{WF}_{\mathrm{bi}}^{12}\left(\left(C_{1} \otimes C_{2}\right) u\right)
$$

there exist operators $H_{i} \in \mathrm{L}^{0}\left(\Omega_{i}\right)$, non-characteristic at $\left(x_{i}, \xi_{i}\right)$, such that $\left(H_{1} \otimes H_{2}\right) u \in \mathcal{C}^{\infty}\left(\Omega_{1} \times \Omega_{2}\right)$.

Proof. By the standard pseudodifferential calculus we can pick $B_{i} \in$ $\mathrm{L}^{-m_{i}}\left(\Omega_{i}\right)$ non-characteristic at $\left(x_{i}, \xi_{i}\right)$. Then the product $B_{i} C_{i} \in \mathrm{L}^{0}$ is non-characteristic at $\left(x_{i}, \xi_{i}\right)$. Proposition 3.10 and the definition of the biwave front set guarantee us the existence of $A_{i} \in \mathrm{L}^{0}$, non-characteristic at $\left(x_{i}, \xi_{i}\right)$, such that $\left(A_{1} \otimes A_{2}\right)\left(B_{1} C_{1} \otimes B_{2} C_{2}\right) u \in \mathcal{C}^{\infty}\left(\Omega_{1} \times \Omega_{2}\right)$. Therefore $H_{i}:=A_{i} B_{i} C_{i}$ fulfils the claim.

\section{Comparison with $S G$ Calculus}

In this section we will compare bisingular calculus with $S G$ calculus. $S G$ calculus is a global calculus obtained from the classical calculus by treating the variables and covariables equivalently by imposing on the symbols similar estimates as in bisingular calculus. These a priori formal similarities lead to interesting similarities in the calculus and in the analysis of singularities. However, the two calculi also differ in important aspects, as we will point out throughout the section.

Definition 4.1. A function $a(x, \xi) \in \mathcal{C}^{\infty}\left(\mathbb{R}^{2 n}\right)$ is called a $S G$ symbol belonging to $\mathrm{SG}^{\mu, m}\left(\mathbb{R}^{n}\right):=\mathrm{SG}^{\mu, m}$ iff for every $\alpha, \beta \in \mathbb{Z}_{+}^{n}$ there exists a constant $C_{\alpha, \beta}>0$ such that

$$
\left|D_{x}^{\alpha} D_{\xi}^{\beta} a(x, \xi)\right| \leq C_{\alpha, \beta}\langle\xi\rangle^{\mu-|\beta|}\langle x\rangle^{m-|\alpha|}
$$

for every $x, \xi \in \mathbb{R}^{n}$. A $S G$ pseudodifferential operator is an operator of the form

$$
A u(x):=\int e^{i x \cdot \xi} a(x, \xi) \hat{u}(\xi) d \xi, u \in \mathscr{S}
$$


and the class of operators with symbols in $\mathrm{SG}^{\mu, m}$ is denoted by $\mathrm{LG}^{\mu, m}$.

A symbol $a \in \mathrm{SG}^{m_{1}, m_{2}}$ is called SG classical if it admits a homogeneous expansion with respect to $\xi$, for $|\xi|>>1$, a homogeneous expansion in $x$, for $|x|>>1$, and the two expansions satisfy certain compatibility conditions, we refer here to [Cor95, ES97] for a precise definition of classical symbols. We limit our attention (in this context) to classical operators, i.e. such that their symbols are $S G$ classical. As usual one proceeds to develop a calculus for these operators. As every classical $S G$ operator $A$ is also classical pseudodifferential operator, it admits a principal symbol $\sigma^{\psi}(A)$, homogeneous in the first variable. In addition, by exchanging the roles of the variables and covariables one obtains a symbol $\sigma^{e}(A)$, homogeneous in the second variable. The two satisfy a compatibility condition, i.e. that there is a third, bihomogeneous symbol $\sigma^{\psi e}(A)$, the leading term of the corresponding expansions of the $\psi$ and $e$-symbols. The principal homogeneous symbol of the operator is then the couple $\left(\sigma^{\psi}(A), \sigma^{e}(A)\right)$ which gives rise to the principal symbol

$$
\operatorname{Sym}_{p}(x, \xi)=\phi_{\psi}(\xi) \sigma^{\psi}(A)+\phi_{e}(x) \sigma^{e}(A)-\phi_{\psi}(x) \phi_{e}(\xi) \sigma^{\psi e}(A),
$$

where $\phi_{*}$ are 0 -excision functions.

So far, this is very similar to the bisingular calculus, but the expansion formula for the symbol of a product is in fact a lot simpler. The operator compositions arising there are not present, and the composition formula is just the one corresponding to the $c^{12}$-term in Theorem [1.7.

This leads to a definition of ellipticity close to our notion of 12-ellipticity, as no such thing as full invertibility of the symbols as operators is needed in the parametrix construction:

Definition 4.2. A symbol $a \in \mathrm{SG}^{\mu, m}$ is $S G$-elliptic iff there exist constants $R, C_{1}, C_{2}>0$ such that

$$
C_{1}\langle\xi\rangle^{\mu}\langle x\rangle^{m} \leq|a(x, \xi)| \leq C_{2}\langle\xi\rangle^{\mu}\langle x\rangle^{m}
$$

when $|x|+|\xi| \geq R$.

With this notion of ellipticity, we have the Fredholm property, i.e. an $S G$ elliptic operator admits a parametrix in the calculus.

Another important aspect to note is that in $S G$ calculus we have

Proposition 4.3. Let $p \in \mathrm{SG}^{m, \mu}\left(\mathbb{R}^{n}\right), q \in \mathrm{SG}^{r, \nu}\left(\mathbb{R}^{n}\right)$. Then the commutator $[P, Q]:=P Q-Q P$ belongs to $\mathrm{LG}^{m+r-1, \mu+\nu-1}\left(\mathbb{R}^{n}\right)$.

while in the bisingular setting the analogon in fact does not hold true, see Corollary 1.7.1 and Example 1.9 which illustrates that the difference stems from an interaction of the $\Omega_{1}$ and $\Omega_{2}$-parts of the operators which is not present in the $S G$ case, where variables and covariables are independent.

The notion of $S G$ calculus can be used to introduce a global analysis of singularities. It turns out that this exhibits interesting similarities with the above analysis of bisingular operators. In the following, we refer to Cor95, CM03; see also CJT13a, CJT13b]. First, we introduce $S G$ characteristic sets and the $S G$ wave front set. 
Definition 4.4 ( $S G$ characteristic sets). Let $A \in \mathrm{LG}^{m_{1}, m_{2}}$. Define the $S G$ characteristic set of $A$ as

$$
\operatorname{Char}_{\mathrm{SG}}(A)=\operatorname{Char}_{\mathrm{SG}}^{\psi}(A) \cup \operatorname{Char}_{\mathrm{SG}}^{e}(A) \cup \operatorname{Char}_{\mathrm{SG}}^{\psi e}(A),
$$

where

$$
\begin{aligned}
& \operatorname{Char}_{\mathrm{SG}}^{\psi}(A)=\left\{(x, \xi) \in \mathbb{R}^{n} \times\left(\mathbb{R}^{n} \backslash 0\right): \sigma^{\psi}(A)(x, \xi)=0\right\}, \\
& \operatorname{Char}_{\mathrm{SG}}^{e}(A)=\left\{(x, \xi) \in\left(\mathbb{R}^{n} \backslash 0\right) \times \mathbb{R}^{n}: \sigma^{e}(A)(x, \xi)=0\right\}, \\
& \operatorname{Char}_{\mathrm{SG}}^{\psi e}(A)=\left\{(x, \xi) \in\left(\mathbb{R}^{n} \backslash 0\right) \times\left(\mathbb{R}^{n} \backslash 0\right): \sigma^{\psi e}(A)(x, \xi)=0\right\} .
\end{aligned}
$$

Definition 4.5 ( $S G$ wave front set). Let $u \in \mathscr{S}^{\prime}\left(\mathbb{R}^{n}\right)$. Define the $S G$ wave front set of $u$ as

$$
\mathrm{WF}_{\mathrm{SG}}(u)=\mathrm{WF}_{\mathrm{SG}}^{\psi}(u) \cup \mathrm{WF}_{\mathrm{SG}}^{e}(u) \cup \mathrm{WF}_{\mathrm{SG}}^{\psi e}(u),
$$

where

$$
\begin{gathered}
\mathrm{WF}_{\mathrm{SG}}^{\psi}(u)=\bigcap_{\substack{A \in \mathrm{LG}^{0,0}\left(\mathbb{R}^{n}\right) \\
A u \in \mathscr{S}\left(\mathbb{R}^{n}\right)}} \operatorname{Char}_{\mathrm{SG}}^{\psi}(A), \\
\mathrm{WF}_{\mathrm{SG}}^{e}(u)=\bigcap_{\substack{A \in \mathrm{LG}^{0,0}\left(\mathbb{R}^{n}\right) \\
A u \in \mathscr{S}\left(\mathbb{R}^{n}\right)}} \operatorname{Char}_{\mathrm{SG}}^{e}(A), \\
\mathrm{WF}_{\mathrm{SG}}^{\psi e}(u)=\bigcap_{\substack{A \in \mathrm{LG}^{0,0}\left(\mathbb{R}^{n}\right) \\
A u \in \mathscr{S}\left(\mathbb{R}^{n}\right)}} \operatorname{Char}_{\mathrm{SG}}^{\psi e}(A) .
\end{gathered}
$$

This notion exhibits the following properties:

Proposition 4.6 (Properties of the $S G$ wave front set). Let $u, v \in$ $\mathscr{S}^{\prime}\left(\mathbb{R}^{n}\right), f \in \mathscr{S}\left(\mathbb{R}^{n}\right)$. Then:

(1) $\mathrm{WF}_{\mathrm{SG}}(u)$ is a closed set and $\mathrm{WF}_{\mathrm{SG}}^{\psi}(u)$ is conical with respect to the variable $x, \mathrm{WF}_{\mathrm{SG}}^{e}(u)$ with respect to the covariable $\xi$ and $\mathrm{WF}_{\mathrm{SG}}(u)$ with respect to both of them independently,

(2) $(x, \xi) \in \mathrm{WF}_{\mathrm{SG}}(u) \Leftrightarrow(\xi,-x) \in \mathrm{WF}_{\mathrm{SG}}(\mathcal{F} u)$ (Fourier Symmetry),

(3) $\mathrm{WF}_{\mathrm{SG}}(u+v) \subseteq \mathrm{WF}_{\mathrm{SG}}(u) \cup \mathrm{WF}_{\mathrm{SG}}(v) ; \mathrm{WF}_{\mathrm{SG}}(f u) \subseteq \mathrm{WF}_{\mathrm{SG}}(u)$,

(4) $\mathrm{WF}_{\mathrm{SG}}(u)=\emptyset \Leftrightarrow u \in \mathscr{S}\left(\mathbb{R}^{n}\right)$ (Global regularity).

Already we see the similarities, but also apparent differences, with the bisingular notion:

- Fourier transformation (i.e. exchange of variables and covariables) corresponds to the exchange of variables $x_{1}$ and $x_{2}$ in Proposition 3.8 .

- The conical properties of the individual components of the wave front sets correspond to the homogeneity properties of the corresponding principal symbol part.

- The global (i.e.) $\mathscr{S}$-regularity is of course due to the fact that $S G$ calculus imposes bounds on the variables also.

Moreover, $S G$ operators satisfy $S G$ microlocalty and microellipticity, i.e. (just as in the bisingular case): 
Proposition 4.7 ( $S G$ Microlocality and $S G$ Microellipticity). Let $u \in \mathscr{S}^{\prime}\left(\mathbb{R}^{n}\right)$ and $C \in \mathrm{LG}^{m, \mu}$. Then we have the double inclusion

$$
\mathrm{WF}_{\mathrm{SG}}(C u) \subseteq \mathrm{WF}_{\mathrm{SG}}(u) \subseteq \operatorname{Char}_{\mathrm{SG}}(C) \cup \mathrm{WF}_{\mathrm{SG}}(C u)
$$

This again stems from an individual double inclusion with respect to each wave front set component. A capital difference lies, however, in the structure of the wave front set. For the components of the $S G$ wave front set have

$$
\mathrm{WF}_{\mathrm{SG}}(u) \subset\left(\mathbb{R}^{n} \times\left(\mathbb{R}^{n} \backslash 0\right)\right) \cup\left(\left(\mathbb{R}^{n} \backslash 0\right) \times \mathbb{R}^{n}\right) \cup\left(\left(\mathbb{R}^{n} \backslash 0\right) \times\left(\mathbb{R}^{n} \backslash 0\right)\right) .
$$

Here, the $\left(\mathbb{R}^{n} \times\left(\mathbb{R}^{n} \backslash 0\right)\right) \ni(x, \xi)$ component corresponds exactly to singularities at finite arguments $x$ with propagation direction $\xi$, the $\left(\mathbb{R}^{n} \times\left(\mathbb{R}^{n} \backslash 0\right)\right)$ component yields the same interpretation in the Fourier transformed space (growth singularities of $u$ become differential singularities of $\hat{u}$ ) and the $\left(\left(\mathbb{R}^{n} \backslash 0\right) \times\left(\mathbb{R}^{n} \backslash 0\right)\right)$ component corresponds to high oscillations present at infinite arguments.

In the bisingular case, new phenomena are present. The 12-component has the classical interpretation, in the sense that it includes all the 'classical' singularities (see Lemma 3.3.1), but the other components lose some amount of localization. This is reflected in the structure of the (1- and 2-components) of the bi-wave front set. In fact,

$$
\begin{aligned}
\mathrm{WF}_{\mathrm{bi}}(u) \subset\left(\Omega_{1} \times \Omega_{2}\right) \times\left(\mathbb{R}^{n_{1}+n_{2}} \backslash 0\right) & =\left(\Omega_{1} \times \Omega_{2}\right) \times\left(\left(\mathbb{R}^{n_{1}} \backslash 0\right) \times\{0\}\right) \\
& \sqcup\left(\Omega_{1} \times \Omega_{2}\right) \times\left(\{0\} \times\left(\mathbb{R}^{n_{2}} \backslash 0\right)\right) \\
& \sqcup\left(\Omega_{1} \times \Omega_{2}\right) \times\left(\left(\mathbb{R}^{n_{1}} \backslash 0\right) \times\left(\mathbb{R}^{n_{2}} \backslash 0\right)\right),
\end{aligned}
$$

where if e.g. for one $x_{1}$ we have $\left(x_{1}, x_{2}, 0, \xi_{2}\right)$ present in the wave front set, all $\left(y, x_{2}, 0, \xi_{2}\right)$ are present in the wave front set. This is due to the fact that bi-ellipticity involves true invertibility, i.e. a non-local requirement.

Another difference arises as follows: the 1 and 2-component can be understood as the boundary faces of the 12-component, whereas in the $S G$ case the $\psi e$-component is interpreted as the corner of the wave front space where the $e$ - and $\psi$-component meet, i.e. the roles as boundaries are interchanged, see Figure 1 .

This has the following consequence:

Example 4.8. Consider the one dimensional case. Following here Example 2.7. in [CM03, there exists a distribution $u(x)=e^{i x^{2} / 2}, x \in \mathbb{R}$, such that $\mathrm{WF}_{\mathrm{SG}}^{\psi}(u)=\emptyset=\mathrm{WF}_{\mathrm{SG}}^{e}(u)$ and $\mathrm{WF}_{\mathrm{SG}}^{\psi e}(u)=(\mathbb{R} \backslash 0) \times(\mathbb{R} \backslash 0)$.

However, there cannot exist a distribution $v \in \mathcal{E}^{\prime}\left(\Omega_{1} \times \Omega_{2}\right), \Omega_{1}, \Omega_{2} \subset \mathbb{R}$, such that $\mathrm{WF}_{\mathrm{bi}}^{1}(v)=\emptyset=\mathrm{WF}_{\mathrm{bi}}^{2}(v)$ and $\mathrm{WF}_{\mathrm{bi}}^{12}(v)=\Omega_{1} \times \Omega_{2} \times(\mathbb{R} \backslash 0) \times(\mathbb{R} \backslash 0)$. This is because $\mathrm{WF}_{\mathrm{bi}}^{12}(v)=\Omega_{1} \times \Omega_{2} \times(\mathbb{R} \backslash 0) \times(\mathbb{R} \backslash 0)$ is an open set, and $\mathrm{WF}_{\mathrm{bi}}(v)$ has to be closed.

Similarly $\tilde{v}=\delta \otimes 1$ has $\mathrm{WF}_{\mathrm{bi}}^{1}(\tilde{v})=\left(\{0\} \times \Omega_{2}\right) \times((\mathbb{R} \backslash 0) \times\{0\}), \mathrm{WF}_{\mathrm{bi}}^{12}(\tilde{v})=$ $\emptyset=\mathrm{WF}_{\mathrm{bi}}^{2}(\tilde{v})$, but there cannot exist a distribution $\tilde{u}$ such that $\mathrm{WF}_{\mathrm{SG}}^{e}(\tilde{u})=$ $\mathbb{R} \times(\mathbb{R} \backslash 0)$ but $\mathrm{WF}_{\mathrm{SG}}^{\psi e}(\tilde{u})=\emptyset$, again due to closedness.

\section{References}

[Bat12] U. Battisti, Weyl asymptotics of bisingular operators and Dirichlet divisor problems, Math. Z. (2012), no. 272, 1365-1381. 


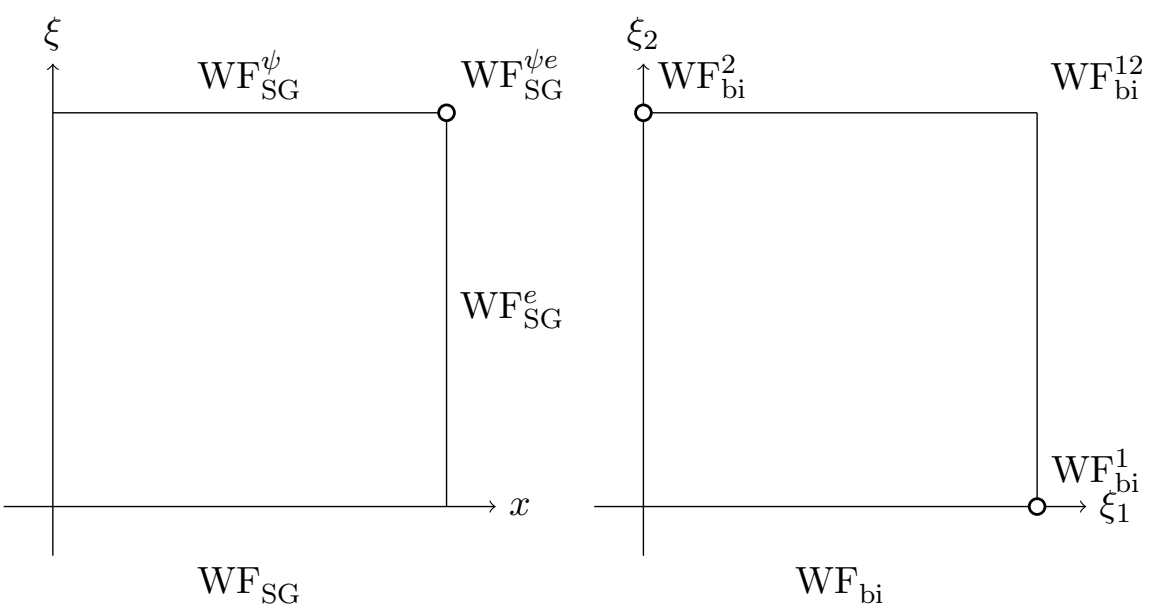

FiguRE 1. A schematic comparison of the components of $\mathrm{WF}_{\mathrm{SG}}$ and $\mathrm{WF}_{\mathrm{bi}}$

[BGPR13] U. Battisti, T. Gramchev, S. Pilipović, and L. Rodino, Globally Bisingular Elliptic Operators, Operator Theory, Pseudo-Differential Equations, and Mathematical Physics, Operator Theory: Advances and Applications, vol. 228, Birkhäuser, Basel, 2013.

[CJT13a] S. Coriasco, K. Johansson, and J. Toft, Global wave-front sets of Banach, Fréchet and Modulation space types, and pseudo-differential operators, J. of Diff. Eq. (2013).

[CJT13b] L Local wave-front sets of Banach and Fréchet types, and pseudodifferential operators, Monatshefte für Mathematik 169 (2013), no. 3-4, 285316.

[CM03] S. Coriasco and L. Maniccia, Wave Front Set at Infinity and Hyperbolic Linear Operators with Multiple Characteristics, Ann. Global Anal. Geom. (2003), no. $24,375-400$.

[Cor95] H. O. Cordes, The Technique of Pseudodifferential Operators, Cambridge University Press, 1995.

[Dud79a] R. V. Dudučava, On the index of bisingular integral operators. I., Math. Nachr. (1979), no. 91, 431-460.

[Dud79b] On the index of bisingular integral operators. II., Math. Nachr. (1979), no. 92, 289-307.

[ES97] Y. V. Egorov and B.-W. Schulze, Pseudo-differential operators, singularities, applications, Operator Theory: Advances and Applications, vol. 91, Birkhäuser Verlagl, Basel, 1997.

[FST98] B.V. Fedosov, B.-W. Schulze, and N.N. Tarkhanov, On the index of elliptic operators on a wedge, J. Funct. Anal. (1998), no. 156, 164-209.

[GH13] D. Grieser and E. Hunsicker, A Parametrix Construction for the Laplacian on $\mathbb{Q}$-rank 1 Locally Symmetric Spaces, Preprint (2013), in http://arxiv.org/abs/1212.3459v2.

[GS94] A. Grigis and J. Sjöstrand, Microlocal Analysis for Differential Operators, An Introduction, Cambridge University Press, 1994.

[Hör83] L. Hörmander, The Analysis of Linear Partial Differential Operators, vol. I, Springer-Verlag, 1983.

[Hör85] The Analysis of Linear Partial Differential Operators, vol. III, Springer-Verlag, 1985.

[Hör91] $\longrightarrow$ Quadratic hyperbolic operators, Microlocal Analysis and Applications (L. Cattabriga and L. Rodino, eds.), Lecture Notes in Mathematics, vol. 1495, Springer Berlin Heidelberg, 1991, pp. 118-160. 
[Mel94] R. Melrose, Spectral and scattering theory for the laplacian on asymptotically euclidian spaces, 1994.

[Mel95] G Geometric scattering theory. stanford lectures, Cambridge University Press, 1995.

[MR06] R. Melrose and F. Rochon, Index in K-theory for families of fibred cusp operators, $K$-theory 37 (2006), pp.25-104.

[NR06] F. Nicola and L. Rodino, Residues and Index for Bisingular Operators, In: $C^{*}$-algebras and elliptic theory, Trends Math., Birkhäuser (2006), pp.187-202.

[Par72] C. Parenti, Operatori pseudodifferenziali in $\mathbb{R}^{n}$ e applicazioni, Ann. Mat. Pura Appl. 93 (1972), 359-389.

[Pil71] V. S. Pilidi, Multidimensional bisingular operators, Dokl. Akad. Nauk SSSR (1971), no. 201, 787-789.

[Pil73] Computation of the index of a bisingular operator., Funkcional. Anal. i Priložen. (1973), no. 7, 93-94.

[Rod75] L. Rodino, A class of pseudo differential operators on the product of two manifolds and applications, Ann. Scuola Norm. Sup. Pisa Cl. Sci. (1975), no. 4, 287-302.

[Rod80] $\quad$ Polysingular integral operators, Ann. Mat. Pura Appl. (1980), no. 124, 59-106.

[Sch87] E. Schrohe, Spaces of weighted symbols and weighted sobolev spaces on manifolds, Pseudo-Differential Operators, Lecture Notes in Math., vol. 1256, Springer, Berlin, 1987, pp. 360-377.

[Shu01] M. A. Shubin, Pseudodifferential Operators and Spectral Theory, second ed., Springer-Verlag, 2001.

[Trè67] F. Trèves, Topological Vector Spaces, Distributions, and Kernels, Academic Press, 1967.

Dipartimento di Matematica "Giuseppe Peano", Università degli Studi di Torino, Via Carlo Alberto 10, 10123 Torino (TO), Italy.

E-mail address: massimo.borsero@unito.it

Mathematisches Institut, Georg-August Universität Göttingen, BunsenStrasse 3-5, D-37073 Göttingen, Germany

E-mail address: rschulz@uni-math.gwdg.de 